Annuaire suisse de politique de développement

2 | 1982

Annuaire Suisse - Tiers Monde 1982

\title{
1. Négociations internationales
}

\section{Hilmar Stetter}

\section{OpenEdition}

Journals

Édition électronique

URL : http://journals.openedition.org/aspd/1116

DOI : $10.4000 /$ aspd. 1116

ISSN : 1663-9669

\section{Éditeur}

Institut de hautes études internationales et du développement

\section{Édition imprimée}

Date de publication : 31 août 1982

Pagination : 13-60

ISSN : 1660-5934

\section{Référence électronique}

Hilmar Stetter, «1. Négociations internationales », Annuaire suisse de politique de développement [En ligne], 2 | 1982, mis en ligne le 19 janvier 2013, consulté le 08 septembre 2020. URL : http:// journals.openedition.org/aspd/1116; DOI : https://doi.org/10.4000/aspd.1116 


\section{NEGOCIATIONS INTERNATIONALES}

\section{CONFERENCE DE L'ONU SUR LES PAYS LES MOINS AVANCES}

A l'unanimité, la conférence de l'ONU sur les pays les moins avancés a adopté un "nouveau programme substantiel d'action" qui répond à une stratégie du développement conçue à l'intention des pays les moins avancés. Alors que les pays en développement demandaient une augmentation de l'aide concrétisée par des engagements financiers et temporels, les pays industrie/s ne se déclarèrent disposés qu'à augmenter leur aide tout en insistant sur la nécessité de modifier la politique intérieure de développement des pays les plus pauvres. La Suisse approuva également le principe d'une augmentation de l'aide. Elle se prononça pour une amélioration qualitative de l'aide, une coordination accrue et demanda qu'on tînt mieux compte des initiatives de la population quant à la détermination des prestations à apporter aux pays en développement les plus démunis.

La conférence des Nations Unies sur les pays les moins avancés s'est tenue à Paris du 1er au 14 septembre 1981. Y prirent part les délégués de cent quarante-deux nations, de trente organisations intergouvernementales ou spécialisées, ainsi que les représentants de soixante organisations non gouvernementales.

Cette conférence a été convoquée suite à une résolution de la CNUCED V de Manille (1979) et à une décision de I'Assemblée générale de l'ONU en décembre 1979. La CNUCED V avait adopté en faveur des pays les plus pauvres un programme d'urgence (pour les années 1979-1981) et un programme d'aide à long terme pour les années 1980.

La conférence de Paris avait pour but de définir concrètement le "nouveau programme substantiel d'action" (NPSA). II s'agissait d'inclure à ce programme les lignes directrices de la politique de développement à adopter par les pays les moins avancés, leurs demandes d'aide internationale et les méca- 
nismes permettant un contrôle constant des mesures adoptées.

Selon les critères de I'ONU, trente et un pays sont actuellement compris dans le groupe des pays les moins développés; il y en a vingt et un en Afrique, huit en Asie, un dans le Pacifique et un en Amérique. En 1981, la population totale de ces pays s'élevait à 282,6 millions d'âmes. La liste s'établit ainsi : Afrique (21) : Bénin, Botswana, Burundi, Cap-Vert, République centreafricaine, Tchad, Comores, Ethiopie, Gambie, Guinée, Guinée-Bissau, Lesotho, Malawi, Mali, Niger, Rwanda, Somalie, Soudan, Tanzanie, Ouganda, Haute-Volta;

Asie (8) : $\quad$ Afghanistan, République arabe du Yemen, Bangladesh, Bhoutan, République populaire du Yemen, Laos, Maldives, Népal;

Pacifique (1): Samoa;

Amérique (1): Haïti.

Les pays sont classés selon les critères suivants, adoptés en 1971 par I'Assem-

blée générale de l'ONU :

- produit intérieur brut inférieur à 100 dollars EU par tête d'habitant (1971);

- part du secteur industriel au produit intérieur brut inférieur à 10\%;

- taux d'alphabétisation jusqu'à 20\%(1).

Les pays les moins avancés diffèrent fortement entre eux à maints égards; ils n'en sont pas moins placés souvent devant les mêmes difficultés de développement qui les distinguent des autres pays en développement, à savoir :

- aucune possibilité pour eux de vaincre leur pauvreté avec leur propre force;

- degré élevé de dépendance du peu de matières premières dont ils disposent et qu'ils exportent massivement;

- leur handicap géographique (Etats insulaires ou sans débouchés sur la mer) est aggravé par l'insuffisance des communications intérieures.

La situation économique de ces pays n'a pas cessé de se détériorer. La baisse de la production agricole et des revenus d'exportations, un faible essor industriel ne leur ont pas permis de surmonter leur pauvreté et n'ont fait qu'aggraver l'écart avec les pays en développement mieux lotis et avec les pays industriels. D'où l'exigence légitime d'une stratégie du développement spécialement conçue à l'intention des pays les plus pauvres.

Les pays en dévelopement ont participé à la conférence avec l'espoir que les pays donateurs augmenteraient le volume de leur aide. Les pays industriels au contraire mirent l'accent sur les modifications à apporter à la politique de développement pratiquée par les pays les plus démunis. Le programme d'action constitue une base de travail qui permettra à l'avenir de contrôler l'aide fournie aux pays les moins avancés et de détecter les modifications de leur situation économique et sociale. La conférence a également permis d'attirer l'attention de l'opinion internationale sur les difficultés des pays les moins avancés. Le programme d'action adopté au terme de la conférence représente le seul compromis possible pour les différentes nations en présence. 
L'aide des pays industriels ne sera distribuée dans les pays en développement les moins avancés ni selon le volume souhaité, ni selon un calendrier précis. En revanche, les prestations promises représentent pour les pays en développement une amélioration des modalités de I'aide (2).

\section{Le "nouveau programme substantiel d'action"}

Dans la première partie du programme d'action, l'énumération des mesures à prendre au plan national, on relèvera que les pays les plus pauvres sont responsables de leur propre politique de développement. A eux de l'adapter à la satisfaction de leurs besoins essentiels. L'agriculture y joue un rôle important, comme une production vivrière suffisante et la mobilisation du secteur rural au service des objectifs du développement national.

Au plan international, des mesures d'ordre financier, technique et touchant aux relations économiques doivent être prises à l'appui de l'effort national. La deuxième partie du programme d'action est consacrée à des mesures économiques internationales.

Les pays donateurs affirment une fois de plus qu'ils souhaitent porter leur aide publique au développement à $0,7 \%$ de leur PNB annuel. Une part croissante de celle-ci devrait être consacrée aux pays les plus pauvres. La plupart des pays donateurs réserveront au cours des années qui viennent $0,15 \%$ de leur PNB à des prestations en faveur des pays les moins avancés. En comparaison avec les cinq dernières années, on parviendrait ainsi à doubler d'ici 1985 le volume général de l'aide publique au développement accordée aux pays les moins avancés.

Les pays en développement sont d'avis que I'aide publique au développement accordée par les donateurs aux pays les moins avancés devrait atteindre au moins $0,15 \%$ du PNB dans la première moitié des années 80 et $0,20 \%$ du PNB dans la deuxième moitié de la même décennie.

En outre, les pays en développement sont d'avis qu'au moins $30 \%$ des programmes d'aide bilatérale et multilatérale devraient aller aux pays les moins avancés. Cela équivaudrait à tripler d'ici 1984 les prestations fournies aux pays les plus pauvres et à les quadrupler d'ici 1990, comme le recommande au demeurant la "stratégie internationale du développement".

Quant aux modalités de l'aide, on s'efforcera par tous les moyens d'accorder des prestations à fonds perdus et d'éviter au moins l'aide liée. On cite aussi la possibilité de financer les frais courants d'un projet, les dépenses locales et certaines postes du budget national.

En ce qui concerne les relations commerciales, on insiste sur le développement et la simplification des préférences douanières et sur la nécessité de supprimer les obstacles tarifaires ou non tarifaires encore existants. On propose en outre un mécanisme financier susceptible de compenser la baisse des recettes d'exportation des matières premières. 
La troisième partie du programme d'action propose de contrôler et de régulariser les mesures prises en faveur des pays les moins avancés, cela au plan national, régional et global, et une meilleure coordination avec les plans nationaux de développement. On recommande aux pays en développement de créer un office central chargé de coordonner l'aide des pays donateurs. Les premières répercussions du programme d'action devront être discutées en 1983 à la CNUCED VI; la CNUCED prendra connaissance en 1985 d'un bilan provisoire (3).

\section{L'attitude de la Suisse}

Dirigée par l'ambassadeur Heimo, la délégation suisse a été parmi celles que les pays en développement ne sont par parvenus à contraindre à une augmentation quantitative de leurs prestations. Dans sa prise de position, elle a cependant laissé entrevoir une progression de son apport en faveur des pays les moins avancés. (En 1980, celles-ci s'élevaient à 140,6 millions de francs, c'est-à-dire à $34 \%$ de l'aide publique au développement et à $0,08 \%$ du PNB. 100,8 millions de francs étaient allés directement aux pays les plus pauvres : aide bilatérale; 39,8 millions de francs d'aide multilatérale constituaient un flux indirect.)

Placée devant une demande de soutien financier accru de la part des pays en développement, la Suisse estime cependant que l'essentiel de ce qui doit être tenté est du ressort des pays en développement eux-mêmes. Les structures internes des pays les moins avancés pourraient bien entraver leurs propres efforts de développement. En consentant de nouvelles prestations, il conviendrait de s'assurer que l'administration locale soutient réellement les initiatives de la population. L'aide étrangère pourrait s'articuler autour de ces considérations.

L'ambassadeur Heimo constata encore qu'à ce jour l'aide internationale n'a pas toujours été suffisamment conforme aux besoins des pays les plus démunis. On ne surmontera pas les difficultés de développement uniquement en contractant des engagements financiers croissants, mais en veillant aussi à une progression qualitative de l'aide. Il est en outre souhaitable de mieux adapter l'aide extérieure à la situation du pays bénéficiaire qui tirera profit d'une meilleure conception, d'une meilleure planification de la coopération au développement entre les pays donateurs et les pays les moins avancés, notamment dans les relations bilatérales; les mesures de contrôle et de coordination prises par la conférence devraient aller dans ce sens. La délégation suisse accorde beaucoup d'importance à la volonté exprimée de mieux coordonner au sein des pays les moins avancés les diverses prestations qu'ils reçoivent.

En ce qui concerne les nouvelles modalités d'aide qui ont été demandées, la Suisse n'y voit aucune difficulté sérieuse. L'aide helvétique en général n'est pas liée et est déjà donnée à fonds perdus. Pour le reste, l'ambassadeur Heimo releva dans son intervention à la conférence que la Suisse envisageait un nou- 
vel élargissement de ses préférences douanières, déjà fort libérales (4).

Le service d'information tiers monde (i3m) a relevé que la conférence s'était également prononcée pour un relèvement de l'aide aux pays les moins avancés et avait envisagé de modifier légèrement la répartition des ressources disponibles. On étudierait aussi un recours plus prononcé à de nouvelles formes d'aide, par exemple des crédits de programmes et des subventions à la balance des paiements, qui mettraient à moins forte contribution la capacité d'absorption limitée des pays les plus pauvres. On voit d'autres possibilités d'accroître l'aide dans le domaine commercial, ne serait-ce qu'en supprimant les obstacles qui entravent les échanges.

\section{CONFERENCE DE L'ONU SUR LES SOURCES D'ENERGIE NOUVELLES ET RENOUVELABLES}

Les pays en développement ont de plus en plus de mal à surmonter leurs difficultés d'approvisionnement en énergie. La conférence a pris pour thème la nécessité de trouver des sources d'énergie nouvelles ou renouvelables. Elle a adopté un programme d'action international. Restait ouverte la question du financement et de l'institutionnalisation de ce programme. La Suisse entend soutenir ce programme d'action et augmenter ses contributions dans ce domaine. Une manifestation parallèle a été organisée en marge de la conférence par les organisations non gouvernementales.

C'est à Nairobi, du 10 au 21 août 1981, qu'a eu lieu la conférence de I'ONU sur les sources d'énergie nouvelles et renouvelables. Y prirent part les délégués de cent vingt-cinq Etats ou organisations internationales et non gouvernementales. La conférence avait été convoquée selon une décision de l'Assemblée générale de l'ONU en décembre 1978.

La situation énergétique mondiale, les difficultés croissantes des pays en développement quant à leur approvisionnement, ont motivé la convocation de cette conférence. Vu leurs besoins croissants, les pays en développement dénués de ressources pétrolières éprouvent le besoin urgent d'exploiter d'autres sources d'énergie, au risque de voir la progression de leurs importations menacer leur équilibre financier et saper dangereusement leur processus de développement.

La conférence avait choisi pour thème restreint la seule étude des quatorze sources d'énergie nouvelles et renouvelables (soleil, géothermie et biomasse; vent, eau, mer; bois de feu, charbon de bois et tourbe; schistes et sables bitumeux; bêtes de trait). On estime à $15 \%$ de la consommation mondiale en énergie la part de ces divers éléments. A eux seuls, le bois et le charbon de 
bois sont pour beaucoup de pays en développement la première source d'énergie (de 60 à $90 \%$ de la consommation totale).

Un projet de programme d'action fut esquissé durant les travaux préparatoires de la conférence; le programme d'action lui-même fut le principal sujet des négociations. Leur but était de préparer les mesures à prendre au plan international pour soutenir l'effort national des pays en développement et de dresser un plan d'initiatives ponctuelles en faveur des sources d'énergie nouvelles et renouvelables. De quoi embrasser tout le potentiel des nouvelles sources d'énergie afin de résoudre le problème dont la solution intéresse particulièrement les pays en développement.

Les pays industriels n'admirent pas les contraintes d'ordre financier ou administratif voulues par les pays en développement et se contentèrent de déclations d'intentions, mais la conférence se déroula tout de même dans une bonne ambiance. Le programme d'action finalement adopté présentait, au chapitre des divers domaines spécialisés, les propositions des pays industrialisés et des pays en développement, ces derniers appuyés partiellement par les pays de I'OPEP et les Etats du COMECON. Les positions du "Groupe des 77" et des EU représentaient les pôles de la divergence, les Etats de la CEE agissant en qualité d'intermédiaires. A part le travail positif fourni pour rédiger ce programme d'action, il y a lieu de relever que la volonté politique de développer les sources alternatives d'énergie a été fructueuse.

\section{Le programme d'action}

Le programme d'action souligne la nécessité d'un passage organisé et pacifique du pétrole et du gaz naturel aux sources alternatives d'énergie; les pays en développement en retireront un avantage économique. Des initiatives sont prévues dans quatre secteurs :

- inventaire et plan d'exploitation du potentiel des sources d'énergie nouvelles et renouvelables;

- exploration, développement et démonstration de ces ressources;

- transfert, adaptation et utilisation de la technologie nécessaire;

- information et formation.

\section{Les résultats de la conférence}

Lors de la conférence, trois chapitres du programme d'action ont été mis en évidence :

- les priorités à respecter lors de la réalisation du programme d'action;

- le financement des mesures proposées;

- la désignation d'un organisme chargé de réaliser le programme d'action.

Parmi les priorités dégagées, il faut signaler une coopération internationale renforcée dans la recherche et le développement des sources alternatives d'énergie. Celles-ci devront d'abord être mises en exploitation au plan national. D'où diverses mesures à prendre au service d'une politique nationale de 
l'énergie; ainsi on peut citer les programmes "Energie pour les régions rurales", par exemple le reboisement, et "Secteurs urbains et industriels".

Au stade de la réalisation du programme, les pays en développement entendent bien ne se laisser imposer qu'un nombre restreint d'objectifs nationaux. Pour leur part, les pays industriels insistent sur les besoins en énergie des régions rurales des pays en développement; ils souhaitent les aider à réaliser des programmes de reboisement. De leur côté, les pays en développement soulignent leurs besoins en énergie spécifiques pour la ville, la campagne et I'industrie.

Pour accélérer la production d'énergies alternatives dans les pays en développement, il faudra augmenter les transferts de technologie, mais les pays "bénéficiaires" refusent de donner des garanties fermes à des projets de "joint-venture". Les pays en développement escomptent que le programme d'action sera financé par une contribution fixe des pays industriels et par la création d'une filiale spécialisée de la Banque mondiale pour l'énergie. Toutes les sources d'énergies alternatives pourraient ainsi être exploitées au plan national, régional et international.

Plusieurs pays industriels ont appuyé cette demande de création d'une filiale de la Banque mondiale, mais pas les EU, qui attendent davantage, dans ce domaine également, d'une initiative privée. Au lieu d'un cadre financier précis, les pays industriels n'ont finalement accepté que de fournir les ressources additionnelles et convenables pour les énergies nouvelles et renouvelables. Ce qui peut signifier que ce financement destiné au secteur énergétique sera simplement prélevé sur d'autres postes. Le financement de la phase préparatoire et de la réalisation du programme d'action sera prochainement budgeté à l'intérieur de l'appareil de l'ONU.

Aucun accord n'ayant été obtenu sur la création d'une institution spécialisée pour poursuivre les travaux de la conférence, c'est le comité ad interim du programme d'action qui prend provisoirement le relais durant un an et qui fera rapport à l'Assemblée générale de I'ONU à fin 1982. Dans l'attente de la décision de l'Assemblée générale, la conférence propose qu'une organisation du système de l'ONU soit chargée de réaliser le programme d'action.

Demeure en suspens la création d'une agence de la Banque mondiale pour les problèmes énergétiques et le financement du programme d'action. Quoi qu'il en soit, la banque mondiale et le PNUD ont été priés de dresser au cours de la présente décennie un inventaire du financement et des investissements nécessaires au développement des sources alternatives d'énergie dans les pays en développement (7).

Lors d'une séance du comité ad interim à Rome (7-18 juin 1982), on a entériné la décision de réaliser le programme d'action et fixé des lignes directrices pour la mise en œuvre du programme et la mobilisation des ressources financières. 


\section{L'attitude de la Suisse}

La Suisse était également représentée, sa délégation étant conduite par l'ambassadeur Heimo, directeur de la DDA. La Suisse peut dans I'ensemble soutenir les objectifs du programme d'action et les mesures proposées. Elle est d'avis qu'il faut utiliser l'aide de manière efficace et veiller dans ce but à la meilleure coordination possible entre les gouvernements et l'appareil de I'ONU. Elle souhaite qu'au moment où le financement du programme d'action interviendra, on retienne en priorité quatre types de projets :

- petits barrages hydro-électriques intégrés;

- bois de feu, charbon de bois et bêtes de trait;

- énergie solaire;

- énergie fournie par le biogaz.

Dans la réalisation du programme, le PNUD joue un rôle important. La Suisse étudie sa participation au "compte spécial de l'énergie" du PNUD, organisme de financement des projets énergétiques. Elle accueille favorablement la proposition de création d'une filiale de la banque mondiale pour l'énergie (8).

II est intéressant de noter qu'en parallèle avec la conférence de I'ONU, quelques organisations non gouvernementales avaient mis sur pied une autre conférence sur le thème de l'énergie où l'on désirait faire fructifier les discussions des délégués officiels. Les représentants des organisations non gouvernementales de cinquante et un Etats remirent leurs rapports aux participants à la conférence et à la presse, misant aussi sur la pression politique locale qui pourrait s'exercer à propos de ces questions après la conférence. Outre les échanges de vues avec les délégués à la conférence, un programme d'action a été élaboré avec les caractéristiques suivantes :

- création des mécanismes internationaux de financement;

- interdépendance des sources alternatives d'énergie et de l'écologie;

- rôle des femmes dans une meilleure utilisation des sources nouvelles et renouvelables d'énergie;

- restrictions de la consommation d'énergie dans les pays industriels;

- fixation d'objectifs précis pour le programme d'action (9).

\section{CONFERENCE DE L'ONU SUR LE DROIT DE LA MER}

Les pourparlers en vue de renouveler la convention de I'ONU sur le droit de la mer ont duré des années et n'ont pas produit le résultat escompté. Certes, on a mis au point un nouveau droit de la mer et des règles pour l'exploitation des ressources minérales des fonds marins, mais l'importance de ces textes demeure incertaine. Les Etats-Unis notamment restent à l'écart et 
de nombreux pays industrie/s occidentaux ont adopté une attitude attentiste. La nouvelle convention sur le droit de la mer crée des zones de souveraineté élargies pour les Etats riverains et un contrôle international de l'exploitation des ressources minérales des fonds marins. En tant qu'Etat industrialisé sans débouché sur la mer, mais doté d'une flotte commerciale opérant sous le pavillon national, la Suisse se montre satisfaite de la teneur de la convention.

En 1982, après neuf ans de négociations, les pourparlers en vue de la conclusion de la convention de I'ONU sur le droit de la mer purent être menés à terme. Du 3 au 28 août 1981, on eut d'abord à Genève la deuxième partie de la 10ème session de cette conférence. Les représentants de cent quarantesix Etats y prirent part. Du 8 mars qu 30 avril 1982, à Genève, eut enfin lieu la 11 ème session qui vit l'accord se réaliser. Les représentants de cent cinquante-sept Etats s'étaient rassemblés dans ce but.

Une initiative maltaise présentée en 1967 servit de base aux négociations, initiative selon laquelle les mers seraient déclarées patrimoine commun de I'humanité qui les exploiterait pour le bien de tous. Les jeunes nations voulaient être davantage associées à cette exploitation de la mer que le progrès technique a intensifiée. Au contraire d'une internationalisation c'est plutôt une nationalisation des mers qui a prévalu, marquée entre autres par l'extension des zones de souveraineté des Etats riverains.

La convention réalisée après de longs pourparlers, influencés à la fin par l'opposition des Etats-Unis, pourra très vraisemblablement entrer en vigueur. Il est cependant difficile de se prononcer de manière définitive sur son importance. Elle perdrait encore plus de sa signification si les Etats européens, encore hésitants, n'y souscrivaient pas, imitant ainsi les Etats-Unis dont on est déjà certain qu'ils resteront à l'écart. Par contre, I'URSS devrait ratifier la convention (10).

Les travaux de la 10ème session furent passablement bloqués par le comportement attentiste des Etats-Unis. La nouvelle administration Reagan avait en effet décidé de revenir sur l'assentiment donnédu temps de Carter au projet d'accord. Ses propositions de modifications concernant notamment les restrictions de la liberté d'exploitation des ressources minérales furent écartées par la majeure partie des Etats en développement au début de la 11 ème session.

\section{La 10ème session : deuxième partie}

Les points importants de la convention réglés lors de la deuxième partie de la 10ème session ont été les suivants :

- Extension à une portée de 12 milles marins des eaux territoriales. Le libre passage des navires étrangers doit y être garanti, dans la mesure où il répond à des préoccupations pacifiques. 
- Création d'une zone économique exclusive de 200 milles marins à l'intérieur de laquelle les Etats disposeront d'un plein et entier droit d'exploitation économique.

- Réglementation de la protection de l'environnement et de l'exploration future de la mer.

Les propositions suivantes ont encore été discutées :

- La zone internationale sera placée sous le contrôle d'une nouvelle autorité internationale qui accordera des licences pour l'exploration et l'exploitation.

- Adoption de règles concernant les transferts de technologie.

La deuxième partie de la 10ème session a envisagé que la discussion de ces propositions pourrait porter désormais sur quatre points principaux :

- le tracé des frontières de la mer;

- la constitution d'une commission préparatoire;

- la participation à la convention de mouvements de libération nationale et d'organisations internationales;

- la protection des investissements pour l'exploitation des ressources minérales des fonds marins qui ont été consentis avant l'entrée en vigueur de l'accord.

La suite des pourparlers a été marquée par la déclaration des Etats-Unis affirmant que l'examen du projet de convention était entré dans une phase décisive, mais n'était pas encore terminé. Les Etats-Unis sont notamment opposés à la partie XI du traité (zone internationale).

De leur côté, les pays en développement se sont basés sur le projet initial d'accord et ont été déçus des hésitations américaines. Ils ne voulaient notamment pas consentir à la modification des points qui avaient déjà été discutés et sur lesquels on s'était mis d'accord. Le porte-parole du "groupe des 77" déclara que les Etats-Unis ne pouvaient pas, sous peine d'évacuer des négociations le principe de la bonne foi, se dresser contre les intentions exprimées par plus de cent cinquante Etats. Si les Etats-Unis pensent pouvoir exploiter les fonds marins hors de tout accord, il y a lieu de leur notifier qu'on ne peut permettre aux uns ce qui reste interdit aux autres.

Sans avoir pu parvenir à ce règlement de l'exploitation des ressources minérales, la conférence a pris quatre décisions importantes:

- délimitation de la zone d'exploitation économique et du socle continental entre Etats situés de part et d'autre de la mer ou ceux dont les côtes se touchent;

- Kingston/Jamaïque sera le siège de la future autorité de l'ONU pour les fonds marins,

- le futur tribunal du droit de la mer de l'ONU aura son siège à Hambourg;

- malgré les questions restées en suspens, le projet d'accord actuel et informel sera désormais le projet officiel.

Après la clôture de la 10ème session, $d$ 'importantes questions attendent 
encore leur solution, c'est-à-dire :

- la participation à la convention des mouvements de libération nationale et des organisations internationales;

- les problèmes de la protection des investissements et

- la partie XI du projet de convention sur l'exploitation des ressources minérales dans la zone internationale (11).

\section{La 11ème session}

Durant la 11 ème session, on aurait dû élaborer le texte définitif de la convention et parvenir à un accord. Les Etats-Unis firent connaître leur attitude attentiste et présentèrent leurs propositions de révision des dispositions sur l'exploitation des ressources minérales ainsi que d'autres modifications. Ils souhaitaient notamment remplacer l'exploitation réglementée des fonds marins par un accord libéral qui eût favorisé leurs propres entreprises.

Les pays en développement et les pays à économie planifiée refusèrent cependant de remettre en question l'essentiel de l'accord proposé et demandèrent la suspension des débats. Ils n'en acceptèrent pas moins l'exigence des principaux pays industriels occidentaux concernant la protection des investissements pour les travaux d'exploration déjà réalisés. Lors des négociations, un groupe de petits pays industriels, dont la Suisse, joua un rôle d'intermédiaire. L'exploitation peut maintenant être préparée par quatre Etats (France, Japon, Inde, URSS) et par quatre consortiums privés sous la direction d'entreprises américaines, ainsi que par l'autorité de l'ONU pour les ressources des fonds marins. Les opérations pourront commencer après l'entrée en vigueur de la convention.

En outre, la position juridique des pays industriels à l'intérieur de la future autorité de l'ONU pour le droit de la mer a été fortifiée. Finalement, les mouvements de libération obtiennent la possibilité de contresigner les textes définitifs de la conférence et de se faire représenter ultérieurement par des observateurs auprès de l'autorité pour le droit de la mer.

Lors du vote sur la convention demandé par les Etats-Unis, cent trente nations se prononcèrent pour la convention, quatre votèrent contre (Etats-Unis, Israël, Turquie, Vénézuela) et dix-sept s'abstinrent, surtout des pays industriels occidentaux et pays à économie dirigée. La Suisse se prononça pour la convention, tout comme la France et le Japon.

La convention doit maintenant être signée à Caracas à la fin de I'année; elle entrera en vigueur après avoir été ratifiée par au moins soixante Etats signataires (12).

Les principales dispositions de la convention de l'ONU sur le droit de la mer La convention de I'ONU sur le droit de la mer comprend entre autres les importantes dispositions suivantes :

- eaux territoriales et droits de navigation : les zones de souveraineté des 
Etats riverains s'étendent à une limite de 12 milles. La navigation pacifique y est possible. Au-delà de ces eaux, le libre passage et le survol sont garantis. Les Etats sans débouché sur la mer ont un droit de libre accès;

- zone d'exploitation économique : à l'intérieur d'une limite de $\mathbf{2 0 0}$ milles, les pays riverains jouissent d'un droit de pêche exclusif;

- socle continental : sous certaines conditions, les Etats riverains sont seuls à pouvoir disposer des ressources minérales situées au-delà de la zone d'exploitation économique jusqu'à une limite de 350 milles (ou même au-delà);

- exploitation des ressources minérales des fonds marins : les gisements de matières premières situés hors de la zone d'exploitation attribuée aux Etats riverains ne peuvent être exploités que sous le contrôle de l'autorité de I'ONU pour les fonds marins. Celle-ci gère sa propre entreprise d'exploitation (UN-enterprise-system) et accorde des licences pour l'exploitation des ressources à des entreprises privées ou d'Etat, ainsi qu'à l'autorité pour les fonds marins. Les ressources minérales des fonds marins sont considérées comme un patrimoine commun de l'humanité;

- les Etats membres sont responsables de la protection de la mer contre les dangers de pollution de l'environnement (13).

\section{Le comportement de la Suisse}

Dirigée par l'ambassadeur Monnier, la délégation de la Suisse, l'un des pays industrialisés sans débouché sur la mer, mais dotée d'une flotte marchande naviguant sous le pavillon national, devait préserver divers intérêts selon les domaines abordés. Pour la flotte marchande, la réglementation d'un libre trafic maritime sur les mers du globe est d'un grand intérêt et les dispositions prévues dans le projet (droit de pavillon, libre accès à la mer, libre navigation) sont satisfaisantes. En ce qui concerne l'exploitation des richesses des fonds marins dans la zone internationale, la Suisse préconise un système interdisant la monopolisation de ces richesses par un nombre restreint de pays. Elle a un intérêt économique certain à participer à l'exploitation grâce aux commandes d'ingénierie ou d'équipement. C'est pourquoi elle s'est opposée à une réglementation trop stricte qui eût compromis de prime abord tout recours à la technologie suisse et pourquoi elle s'est prononcée contre un trop généreux transfert de technologie (14).

L'hiver passé, entre les deux sessions, eut lieu une rencontre des représentants de huit pays industriels et de représentants de l'industrie soucieux de coordonner leurs législations nationales en dehors des pays en développement et de la convention de I'ONU sur les droits de la mer. Cette mini-convention des Etats qui disposent des capitaux et de la technologie indispensables à I'exploitation des richesses sous-marines devrait mener à l'établissement d'une base légale commune afin qu'on puisse se lancer dans une exploitation requérant de larges investissements (15).

L'Union soviétique a également publié un décret sur l'exploitation des ri- 
chesses des fonds marins au-delà du socle continental. Ce texte fait foi jusqu'à l'entrée en vigueur de la convention sur le droit de la mer (16).

\section{CONFERENCE SUR LA REVISION DU DROIT INTERNATIONAL DES BREVETS}

Au cours de la session de l'an passé de la conférence sur le droit des brevets, les pays en développement qui cherchaient à obtenir une révision du droit des brevets ont obtenu la possibilité d'introduire des licences non volontaires exclusives. Cette proposition se heurte à une vive résistance des pays industriels; les Etats-Unis s'y opposent fondamentalement. Divisés entre eux, les pays industriels estiment possible un échec de la révision. La Suisse appartient au groupe actif des pays industriels qui désirent affaiblir le plus possible la révision. La prochaine session aura lieu en octobre 1982.

Du 28 septembre au 24 octobre 1981 s'est tenue à Nairobi la deuxième session de la conférence diplomatique sur la révision de la Convention de Paris sur la protection de la propriété industrielle. C'est I'OMPI qui l'avait convoquée. $Y$ prirent part les représentants de soixante-huit Etats ainsi que des observateurs de seize organisations inter-étatiques ou non étatiques. Une première session avait eu lieu à Genève en 1980, une troisième session de la conférence sur le droit des brevets a été fixée à Genève en octobre 1982 (17).

A I'heure actuelle, $85 \%$ des brevets exploités dans les pays en développement se trouvent en mains étrangères, leurs détenteurs se recrutant principalement aux Etats-Unis, en République fédérale allemande, en France, en GrandeBretagne et en Suisse, les "cinq grands" dans le domaine des brevets. Dans la situation actuelle, ce n'est pas tant la propriété des brevets par des étrangers qui est en cause, mais l'utilisation qui en est faite.

La majorité des brevets déposés dans les pays en développement - c'est-àdire 35\% - ne sont en effet pas utilisés. De plus ils appartiennent pour la plupart à quelques entreprises transnationales des "cinq grands". Généralement, un brevet ne protège pas seulement un produit ou une technique de fabrication, mais aussi son commerce et son importation. Selon les déclarations des pays en développement, les brevets déposés mais inutilisés exercent une influence négative sur leur croissance économique et freinent leur industrialisation (18).

La conférence a pour but d'adapter à la situation économique et technologique des pays en développement les dispositions sur la protection minimale des brevets, modèles et marques, et celles qui concernent la lutte contre la concurrence déloyale. Un renforcement des sanctions favoriserait l'exploita- 
tion industrielle des inventions brevetées dans les pays en développement. La révision prévue souligne certaines innovations à introduire dans le droit des brevets. Alors que les fournisseurs de technologie mettaient jusqu'ici I'accent sur la protection des droits de monopole du détenteur du brevet, les pays en développement se préoccupent surtout de l'exploitation artisanale et industrielle des brevets.

La base de la révision est un travail d'experts déjà fort élaboré et datant de 1975. Ce texte prévoit des contraintes dans I'exploitation des brevets et des sanctions accrues sous forme de licences non volontaires exclusives ou de caducité du brevet en cas d'exploitation délaissée ou insuffisante.

\section{Les délibérations de Nairobi}

Une refonte de l'art. 5A de la Convention de Paris était au premier plan des délibérations de Nairobi. Jusqu'ici, ce texte gênait considérablement la procédure contre le refus de tirer parti d'une invention. Dans sa forme actuelle, la nouvelle version "fixe les conséquences juridiques dans les cas où le brevet n'est pas trop peu ou abusivement utilisé et elle prévoit des mesures spéciales en faveur des pays en développement. Ceux-ci ont le droit de prévoir dans leur législation l'octroi de licences non volontaires exclusives; avec des dispositions restrictives cependant, à savoir que la licence ne peut pas être accordée avant un délai de trente mois à compter de la date d'octroi du brevet et ne peut l'être qu'en cas d'abus de brevet (le simple fait de ne pas utiliser son droit de patente ne peut donc être taxé d'abus); en outre, l'exclusivité ne peut pas excéder une durée maximale de quatre ans et demi. Enfin, les pays en développement peuvent décréter la caducité et le retrait du brevet s'il est officiellement établi, après un délai d'attente de cinq ans à partir de l'octroi du brevet, qu'une licence non volontaire exclusive ne suffit pas à garantir l'exploitation industrielle de l'invention sur le territoire national" (19).

Inspirée pour les points essentiels par les pays en développement, cette révision s'est heurtée à une ferme résistance des représentants des pays industriels. Ceux-ci ont tenté d'abord, au début de la conférence, d'affaiblir les revendications présentées, sans parvenir pour autant à unifier leurs propositions de révision. Un groupe de six pays industriels de peu de poids dans le domaine des brevets (Australie, Canada, Nouvelle-Zélande, Portugal, Espagne, Turquie) se prononça contre des droits spéciaux à reconnaître aux pays en développement. Ce groupe réclamait une application universelle uniforme du droit des brevets, mais en y faisant figurer les propositions de révision présentées par les pays en développement. Cela, afin de profiter également des avantages recherchés par les pays en développement.

Après constatation des points controversés et divers débats en sous-commission, les représentants des pays industriels parvinrent à obtenir des concessions supplémentaires de la part des pays en développement. Le détenteur d'un brevet inexploité ne peut maintenant plus le voir frappé de caducité et d'une décision de retrait s'il n'a pas eu la possibilité de se justifier. L'octroi 
de licences non volontaires exclusives est soumis aux mêmes conditions.

Après les délibérations que nous relatons, une question reste ouverte : universalité du droit des brevets ou éventuels droits spéciaux à accorder aux pays en développement et aux pays industriels les plus faibles économiquement.

Ces propositions de révision ne sont qu'une partie de la refonte totale envisagée et ne seront soumises au vote qu'à la fin de la conférence. Cependant, le représentant des Etats-Unis a déjà fait savoir qu'il s'opposait au résultat actuel des négociations (20).

\section{Le rôle de la Suisse}

La délégation suisse était dirigée par P.Braendli, directeur de I'OFPI (Office fédéral de la propriété intellectuelle); elle comprenait divers membres de cette administration et, entre autres, un représentant du Vorort. Le chef de la délégation suisse était en même temps le porte-parole des pays industriels. La Suisse fut également représentée dans toutes les négociations intermédiaires et joua ainsi un rôle important lors du déroulement de la conférence.

Au cours des délibérations, la Suisse fut avec les Etats-Unis, la principauté du Liechtenstein et le Japon I'un des quatre pays qui refusèrent d'accepter I'entrée en matière sur les propositions de révision présentées par les pays en développement. Elle se porta également en tête de la première contreoffensive des pays industriels. La Suisse ne cessa de se prononcer pour le maintien de la clause justificative en faveur des détenteurs de brevets et contre les licences non volontaires exclusives. Sur d'autres points (caducité du brevet, délais), la Suisse se révéla comme un interlocuteur coriace ou s'aligna régulièrement sur les propositions du groupe majoritaire des pays industriels (21).

Avant le début de la conférence, il y eut une interpellation parlementaire qui permit au Conseil fédéral, dans sa réponse, d'esquisser entre autres les objectifs poursuivis par la délégation suisse : "La Suisse est l'un des Etats initiateurs de la Convention de Paris sur le droit d'association, en 1883, et elle en a toujours été membre. Figurant parmi les plus notables dispensateurs de technologie, elle considère avec un intérêt tout particulier l'élaboration adéquate d'une protection industrielle internationale. Les multiples relations commerciales de notre pays et la recherche constante de nouveaux marchés d'exportation nous invitent par ailleurs à élargir le cercle des membres de la Convention en y faisant entrer les Etats du Tiers Monde.

Notre pays a donc intérêt à ce que le niveau de protection juridique atteint par la révision, lors des six précédentes adaptations de la convention, soit maintenu dans toute la mesure du possible et il œuvrera dans ce but. II ya lieu de s'opposer à des exigences déplacées et susceptibles de provoquer une érosion du droit de protection industrielle, notamment du droit des brevets. Par ailleurs, des adaptations aux besoins particuliers des pays en développement, notamment dans le domaine du droit des brevets, ne sont pas exclues 
et répondent aux impératifs de notre politique de développement. Ces adaptations doivent être conçues de manière à ne pas gêner le flux souhaitable et le développement de la technologie tout en réalisant un juste équilibre entre les détenteurs d'un droit de protection juridique, d'une part, et les utilisateurs, d'autre part.

La Suisse se préoccupe également de sauvegarder le caractère universel de la Convention de Paris; car seule cette universalité garantit l'aménagement à l'échelle mondiale des droits de protection industrielle. Un traitement spécial en faveur de certains pays ou groupes de pays doit donc rester l'exception et ne pas substantiellement compromettre le caractère universel de l'accord'" (22).

Les résultats obtenus lors de cette deuxième session de Nairobi furent refusés à I'unanimité par les associations professionnelles concernées dans les pays industriels. Ces associations préconisent un retrait des positions actuelles, propositions qui vident, selon elles, le droit des brevets de son sens (23).

Lors d'une réunion préparatoire du "Groupe B" en juin 1982, à Munich, les intérêts divergents des Etats membres restèrent inamovibles. II semble maintenant qu'on veuille s'en tenir aux jalons posés à Nairobi. Les Etats scandinaves insistent sur ce point, alors que les Etats-Unis persistent dans leur opposition. Dans les coulisses, on entend des délégués et des spécialistes ouesteuropéens du droit des brevets affirmer que la révision est sur une mauvaise voie. A longue échéance, selon eux, les pays en développement $n$ 'auraient aucun avantage à cette révision et les négociations devraient recommencer à zéro (24).

\section{AUTRES CONFERENCES}

\subsection{Conférence de la FAO}

Opposition à la 21ème conférence de la FAO : cinq pays industriels, qui sont aussi les principaux pourvoyeurs de fonds de l'organisation, ont voté contre l'adoption du budget. I/s désiraient exprimer ainsi leur critique à des dépenses croissantes mais pas toujours efficientes de la FAO, alors qu'eux-mêmes doivent introduire des mesures d'économie au niveau national. Pas question cependant de réduire l'aide alimentaire ni le soutien à l'agriculture. La Suisse a voté contre le budget.

La 21ème conférence de la FAO a été organisée à Rome du 7 au 26 novembre 1981 , où elle se réunit tous les deux ans. Cent cinquante-deux Etats membres 
y étaient représentés, donc cinq récemment admis. Les thèmes majeurs des débats portaient sur la situation globale de l'alimentation et de l'agriculture, les lignes de force d'une politique agricole et la gestion de la FAO.

Le débat général et les travaux de la première commission appelée à examiner la situation de l'alimentation et de l'agriculture dans le monde se déroulèrent sans trop de controverses, tout comme les questions juridiques et administratives traitées par la troisième commission. Par contre, les réunions de la deuxième commission furent placées sous le signe de la controverse. Par ailleurs, le directeur général de la FAO, E.Saouma, fut confirmé dans ses fonctions pour une nouvelle période de six ans.

\section{La critique du budget}

Les discussions de la deuxième commission portèrent surtout sur l'augmentation du budget administratif et l'organisation interne des programmes de la FAO. La FAO proposait un budget ordinaire de deux ans devisé à 368 millions de dollars $E U$, ce qui équivaut par rapport à la période écoulée à une augmentation de $5,8 \%$ en termes réels. Presque sans exception, les pays en développement approuvèrent ce nouveau budget, alors que la plupart des pays industrialisés critiquaient une telle augmentation.

Au vote, le budget fut approuvé par cent vingt oui, cinq non exprimés par des pays industriels, dont la Suisse, et neuf abstentions de pays industriels de nouveau, à une exception près. Les cinq pays rejetants (Etats-Unis, Japon, République fédérale d'Allemagne, Grande-Bretagne et Suisse) contribuent pour $53,65 \%$ au financement de I'organisation. La Suisse fit une déclaration au nom des cinq pays ayant voté non. Quatre Etats qui s'étaient abstenus de voter s'associèrent à cette déclaration.

Cette déclaration souligne qu'en refusant le budget, on n'a pas dit non à I'amélioration de la situation alimentaire des pays en développement et qu'on ne méconnaît pas le rôle important joué par la FAO. On a cependant estimé ne pas pouvoir consentir à de telles augmentations budgétaires au plan international à l'heure où il s'agit de faire des économies à l'échelon national. Même en cas de croissance zéro de son budget, en admettant tout au plus une compensation de l'inflation, la FAO pourrait s'acquitter de manière satisfaisante de ses tâches essentielles. Pour y parvenir, il lui faudrait rationaliser son appareil administratif, repenser ses priorités et laisser tomber les programmes superflus ou désuets. L'augmentation du budget administratif ne contribue pas automatiquement - et au surplus que très marginalement à l'amélioration de l'alimentation dans les pays en développement.

Finalement, sur proposition mexicaine, la conférence a adopté une résolution sur les semences, patrimoine génétique de l'agriculture ("Gen-Resources").. Elle demande que la $F A O$ élabore un projet de convention qui règle l'accès et les échanges de semences. La résolution demande en outre qu'une étude soit entreprise pour la création de banques de semences agricoles sous les auspices de la FAO. 
La délégation suisse était conduite par le directeur de l'Office fédéral de l'agriculture, M.Piot. Elle tint à réaffirmer son soutien aux buts de la FAO, bien que refusant le budget; la FAO "ayant une tâche très importante à remplir dans le domaine de l'alimentation et particulièrement dans la lutte contre la faim dans le Tiers Monde..." (25). Au cours de discussions ultérieures, elle ne se montra nullement hostile à une décentralisation plus poussée de la FAO, mais certains points du programme lui paraissent encore peu clairs. Elle pense également qu'une décentralisation hâtive ne se ferait pas dans les meilleures conditions.

Quant au "Programme de coopération technique", la Suisse partage I'avis d'autres pays industriels, à savoir que ce programme doit être maintenu, mais pas poussé plus avant. En ce qui concerne les programmes d'actions spéciales qui n'émergent pas du budget ordinaire mais dont la Suisse est l'un des six principaux fournisseurs de fonds, la délégation suisse regrette leurs évaluations insuffisantes et fait dépendre la continuation de son soutien financier d'une meilleure direction et d'un meilleur contrôle des projets.

Le système d'évaluation de la FAO fut également en butte aux critiques des pays industriels, car sa valeur est purement interne. La Suisse serait disposée à seconder le secrétariat de la $\mathrm{FAO}$ dans la mise au point de systèmes d'évaluation complémentaires (évaluations tripartites, par exemple) et, à certaines conditions, se déclare prête à fournir une contribution substantielle à l'installation de cet appareil d'évaluation.

Dans sa prise de position sur les "Gen-Resources" , la délégation suisse fit savoir qu'une banque de semences $F A O$ et une convention internationale ne lui paraissaient pas indispensables, mais qu'elle souhaitait une meilleure coordination entre les banques de semences existantes (26).

\subsection{Conférence de l'ONU sur l'environnement}

Ces dernières années, malgré des efforts de sensibilisation à l'échelle mondiale, la dégradation de l'environnement naturel du fait de l'homme a encore progressé. Tel est le premier bilan dressé par le Programme des Nations Unies pour l'environnement (PNUE). Alors que les pays en développement recherchent une solution dans un cadre global, les délégués à la conférence n'ont pu se mettre d'accord que sur le renforcement de certaines activités figurant au programme. La Suisse a présenté en son nom quelques propositions à la conférence.

C'est au siège du PNUE, à Nairobi, qu'a eu lieu du 10 au 18 mai 1982 une 
réunion extraordinaire de ce Programme des Nations Unies pour l'environnement. Cent cinq Etats y étaient représentés et, parmi eux, la Suisse dont la délégation était dirigée par le Conseiller fédéral Hürlimann.

Le PNUE a été fondé en 1972, lors de la première conférence ministérielle de I'ONU sur la protection de l'environnement, à Stockholm. C'est le seul organisme au sein duquel gouvernements et organisations internationales peuvent se retrouver régulièrement pour traiter des problèmes posés par la protection de I'environnement à l'échelle mondiale. Le PNUE ne réalise pas de projets lui-même; il incite leur création, participe à leur financement et surveille leur marche.

L'activité du PNUE se développe dans trois domaines différents : dans le cadre de ce qu'on appelle le "Plan Vigie", il contrôle l'état global de l'environnement. La protection de l'environnement proprement dit se réalise grâce à des programmes de protection des mers régionales, par exemple, ou de la lutte contre I'avance des steppes. Le PNUE accorde enfin une place importante à certaines mesures complémentaires telles que la formation et l'éducation dans le domaine de l'environnement. Nombre d'activités du PNUE visent les pays en développement.

La session extraordinaire du PNU à Nairobi fournit I'occasion de passer en revue les dix années d'activité après Stockholm et d'envisager l'avenir. Depuis que le PNUE a commencé ses travaux, la sensibilisation aux problèmes de l'environnement a progressé un peu partout. Le rapport entre environnement et développement a été établi. Les buts fixés dans le programme d'action 1972-1982 n'ont été réalisés que partiellement, compte tenu du rythme croissant des dommages subis par I'environnement. La dégradation de l'environnement naturel du fait des activités humaines a continué de s'aggraver. Si cette tendance se maintient, la situation de l'environnement se détériorera. Aujourd'hui comme hier, les forêts, les sols fertiles et les eaux sont tout aussi menacés que l'atmosphère, la faune et la flore.

$\mathrm{Vu}$ l'attention accrue que requièrent les problèmes d'un environnement toujours plus menacé, les pays en développement réclament une fois de plus un meilleur financement du programme du PNUE; ils songent notamment à leurs propres besoins et ils se prononcent en faveur de la création d'un nouvel ordre économique international (NOEI).

Les pays industriels veulent se contenter d'une déclaration d'intentions sans engagements et sans contracter de grandes obligations financières. Ils soulignent que les pays en développement sont les premiers responsables de la résolution de leurs problèmes d'environnement et se disent prêts à promouvoir des projets de développement conçus en tenant mieux compte des données écologiques. Le PNUE continuera à catalyser les travaux sur la protection de l'environnement et ne deviendra pas opérationnel. Si le programme d'action 1972-1982 n'a été réalisé que partiellement, c'est parce que les Etats n'ont pas pu exprimer politiquement leur volonté d'aboutir, à cause 
du manque d'argent et du fait de difficultés de coordination, a-t-on pu lire dans la déclaration finale. Pour améliorer la coordination, la création d'un système "SWMTEP" a été prévue pour donner toutes les informations possibles et imaginables sur les travaux touchant à l'environnement, entrepris par les organisations à l'intérieur et à l'extérieur du système de I'ONU.

Les pays en développement seront invités à faire leur part. Une croissance démographique élevée, des modèles unilatéraux de développement et le saccage des ressources naturelles engendrent les problèmes d'environnement. Seul un NOEI peut venir à bout de la pauvreté et de la surconsommation. La solution des problèmes d'environnement est une tâche mondiale.

Si la déclaration finale n'engage pratiquement personne, un autre document a été rédigé sous forme de programme et ce texte contient des exigences concrètes. Le PNUE doit propager une meilleure information sur les problèmes de l'environnement à l'échelon régional et international; on insiste pour que des lois nationales soient promulguées en conséquence, on exige aussi un contrôle des exportations de pesticides et d'autres produits qui, interdits dans les pays industriels, sont exportés dans les pays en développement.

Le conseil d'administration est invité à préparer les modalités de la création d'une "commission indépendante". Cette commission devrait élaborer une stratégie pour l'environnement jusqu'à I'an 2000. Cette proposition de la session extraordinaire du PNUE devra être examinée par l'Assemblée générale de I'ONU (27).

\section{Les propositions de la Suisse}

Lors de la session extraordinaire, la Suisse s'est inspirée de la loi sur l'environnement en voie d'élaboration pour affirmer que les préoccupations d'ordre jusqu'ici national devraient trouver leur expression juridique à l'échelle internationale. La protection de l'environnement devrait susciter des mesures avant tout préventives; au plan international, le principe de causalité devrait pouvoir être invoqué contre les responsables d'un comportement nuisible au milieu vital. Cependant les postulats relatifs à la protection de l'environnement ne devraient être acceptés qu'en fonction des moyens financiers limités dont on dispose. La Suisse n'est enfin pas opposée à la création d'une commission de protection de l'environnement (28). Sa contribution au budget global du PNUE de 30 millions de dollars EU s'élève à 1 million de francs. La Suisse était représentée à l'assemblée du conseil d'administration du PNUE, qui a suivi la conférence, car elle y a été élue pour trois ans en 1981 (29).

En marge de la conférence, un symposium international a rassemblé des associations de protection de l'environnement de quarante-cinq pays. La Suisse n'y était pas représentée. Les associations de protection de l'environnement des pays en développement ont exprimé l'avis que le plus grand danger pour I'environnement de leurs pays venait des pays industriels, gros consommateurs 
de ressources naturelles importées des pays en développement.

\subsection{Assemblée générale de l’OMS}

L'OMS veut intervenir dans le domaine des brevets et développer la fourniture de médicaments essentiels aux populations. Elle entend aussi travailler au respect du "code international de commercialisation des substituts du lait maternel". Ces prises de position résultent de sa 35ème assemblée générale. La Suisse a accueilli favorablement ces activités.

La 35ème assemblée générale de l'OMS a eu lieu à Genève du 3 au 14 mai 1982. En premier lieu, I'adoption du programme de travail de I'organisation pour les années 1984-1989. Ce programme sert de base à la stratégie "Santé pour tous jusqu'à I'an 2000". II s'agit essentiellement de pourvoir à la santé de la population de tous les pays. L'assemblée a pris ensuite d'autres décisions importantes touchant à la politique de la santé publique.

La politique de l'OMS concernant les brevets, les médicaments et le code international de commercialisation des substituts du lait maternel

L'OMS développera ses activités dans le domaine des brevets en rapport avec la santé; elle entend réaliser son programme de travaux sur les médicaments essentiels et promouvoir l'application du code international de commercialisation des substituts du lait maternel. En ce qui concerne les brevets, I'OMS va surtout encourager la recherche là où elle est nécessaire. Elle peut le faire elle-même ou mandater des institutions publiques ou privées. L'organisation fera l'acquisition de brevets, de certificats d'invention ou prendra des participations dans les technologies sanitaires brevetables. Elle espère ainsi développer des technologies nouvelles d'intérêt commun. Les résultats obtenus seraient accessibles à tous.

En faisant l'acquisition de certains brevets, l'OMS espère inciter l'industrie pharmaceutique à pousser la production de médicaments utiles. Dans ces cas-là, I'OMS garantirait aux pharmaceutiques de l'invention une large part du marché. L'organisation veillerait à ce que le prix de ces médicaments reste accessible aux populations des pays en développement. Un premier médicament inscrit au programme pourrait être un vaccin contre la malaria (30).

L'OMS va également réaliser un programme de ravitaillement de la population des pays en développement portant sur les deux cents médicaments et vaccins essentiels dont elle a fait dresser la liste. L'OMS s'engage par une résolution 
à soutenir les plans nationaux de fourniture de ces deux cents médicaments essentiels aux populations. D'autres institutions, ainsi que l'industrie pharmaceutique et les organisations non gouvernementales, sont invitées à coopérer à la réalisation de cet objectif.

A ce jour, les pays en développement ont été principalement approvisionnés par l'industrie pharmaceutique des pays industriels occidentaux. Le programme mis au point entend aussi développer la production locale des médicaments.

L'association internationale de l'industrie pharmaceutique (AIIP) a déjà proposé à I'OMS de vendre des médicaments essentiels aux pays en développement à des prix hors commerce. L'organisation de consommateurs AIS (Action internationale pour la santé) reproche à cet égard à l'industrie pharmaceutique de vendre ses médicaments à des prix exagérés aux pays en développement et demande à l'OMS de mettre au point un code de commercialisation des médicaments (31).

Dans une troisième résolution, I'OMS prend une position à l'égard du code de commercialisation des substituts du lait maternel adopté en 1981 (code international de commercialisation des substituts du lait maternel). Quelques pays seulement l'ont ratifié. L'OMS demande aux Etats membres d'insérer le code dans leurs législations. L'organisation continuera à encourager l'allaitement maternel et constate que de nombreux pays encouragent l'allaitement (32).

Dans le camp de l'industrie alimentaire, on notera qu'à ce jour Nestlé a mis au point des directives internes qui prescrivent l'application du code de I'OMS et en font l'un des objectifs de sa politique commerciale. Toutes les filiales de Nestlé qui mettent en vente des substituts du lait maternel doivent respecter les dispositions du code. Les représentants des associations de consommateurs pensent qu'il s'agit plutôt d'une concession d'ordre tactique que d'une modification réelle de sa stratégie de marketing. Nestlé n'aurait accepté le code que parce que ses méthodes de vente étaient sujettes à une critique croissante. Les directives de Nestlé s'écarteraient des recommandations du code sur certains points (33). D'autre part, l'association suisse des fabricants de produits alimentaires a aussi publié des directives à l'intention de ses adhérents.

En 1981, la Suisse acceptait le code. Répondant à une simple question, le Conseil fédéral a fait savoir le 14 décembre 1981 "qu'il n'était cependant pas question d'insérer la totalité du code dans notre législation, car ce texte heurte le principe de la liberté du commerce et de l'industrie".

Pour que les dispositions essentielles du code puissent aussi être appliquées en Suisse, l'Office fédéral de la santé publique a désigné un groupe de travail chargé de proposer les mesures adéquates. L'allaitement au sein devra être . notamment encouragé (34). 


\subsection{Commission des Nations Unies sur les sociétés transnationales : code de conduite}

Les consultations au sujet du code de conduite pour les sociétés transnationales n'ont guère progressé. Le groupe de travail des Nations Unies n'est pas parvenu à terminer ses travaux selon le plan prévu et de nombreux paragraphes du code restent en blanc. La commission des Nations Unies pour les entreprises transnationales décidera de la marche à suivre.

La commission des Nations Unies pour les sociétés transnationales a tenu sa 7ème session à Genève, du 31 août au 14 septembre 1981. Le président fit remarquer que l'élaboration du code n'avait que peu progressé, bien que le document fût rédigé aux deux tiers. Les travaux entamés devant porter sur une période plus longue que celle qui avait été prévue, on renouvela d'un an le mandat confié au groupe de travail intergouvernemental. La commission s'occupa aussi de coopération technique, du flux des informations dépassant les frontières nationales, d'un système intégré d'informations et de l'activité du Centre des Nations Unies pour les sociétés transnationales.

Au demeurant, la commission accepta une résolution du "Groupe des 77" qui demande aux gouvernements de prendre des mesures pour mettre fin aux activités des sociétés transnationales en Afrique du Sud. Ces activités devraient par ailleurs faire l'objet d'un rapport et de débats publics. Cette résolution fut acceptée par vingt-trois voix contre cinq, dont celle de la Suisse, et cinq abstentions. Pour finir, la commission remercia la Suisse d'avoir apporté sa contribution volontaire au programme "Coopération technique du Centre des Nations Unies pour les sociétés transnationales".

Durant I'année 1981-1982, le groupe de travail des Nations Unies sur les transnationales tint trois séances, sans parvenir à parachever le code qu'il prépare. Le Suisse Louis von Planta, président du Vorort et délégué du conseil d'administration de Ciba-Geigy, fut nommé membre "expert-conseiller" du groupe de travail intergouvernemental. Ce groupe de conseillers comprend seize personnalités représentant des entreprises, des syndicats et des universités. A I'ouverture des débats les points suivants étaient à traiter :

- préambule;

- règles de conduite pour l'activité des sociétés transnationales;

- devoirs des pays d'accueil;

- réglementation de la coopération entre les Etats et de l'application du code.

Ce sont les travaux concernant les règles de conduite des sociétés qui ont fait le plus de progrès. On a codifié :

- le respect de la souveraineté nationale et l'observation des lois du pays d'accueil en concordance avec les priorités établies et les objectifs de 
I'essor économique et du développement;

- une attitude loyale envers les valeurs et les ambitions sociales et culturelles;

- le respect des droits de l'homme et des libertés fondamentales;

- la non-immixtion dans les affaire politiques internes et les relations entre Etats;

- le refus des pratiques commerciales entachées de corruption.

Des divergences d'opinions subsistent concernant le traitement des sociétés transnationales par les pays d'accueil. II s'agit des points où la législation nationale des pays en développement se trouve limitée par d'autres dispositions. Dans l'optique des pays industriels, "on ne dispose d'aucune règle satisfaisante d'indemnisation et l'accord sur le libre recours au droit et aux tribunaux fait défaut dans le cas des investissements contestés... Le libre transfert des paiements courants et du produit des liquidations d'entreprises n'est pas non plus réglé et il en va de même des cas où se trouve enfreint le principe du traitement de faveur appliqué au pays d'accueil quand il s'agit d'un pays en développement" (35).

Une autre question est restée sans réponse : les pays industriels insistent pour que le code ne soit pas contraignant en droit et pour que les entreprises des pays à économie dirigée soient mises au rang des sociétés transnationales (36). Le groupe de travail a transmis à la commission des Nations Unies le projet de code partiellement achevé. La commission décidera de la marche à suivre lors de sa réunion annuelle de 1982, à fin août (37).

\section{CNUCED}

\subsection{Conseil de la CNUCED : 23ème et 24ème sessions}

Pour la première fois, la CNUCED a publié un rapport sur "Commerce et développement", présentant ainsi sur l'économie mondiale le résultat de ses analyses. Le conseil de la CNUCED a également discuté du protectionnisme et des ajustements structure/s. II a décidé que la CNUCED VI de juin 1983 se réunirait à Belgrade. La Suisse porte un jugement réservé sur le rapport et préconise d'autres buts à sa politique commerciale.

La discussion du "Rapport de la CNUCED sur le commerce et le développement en 1981", ainsi que la désignation du lieu de réunion de la CNUCED VI ont dominé la 23ème session du conseil de la CNUCED du 28 septembre au 6 novembre 1981. 


\section{La 23ème session}

Dans son rapport sur le commerce et le développement, publié en 1981, la CNUCED constate qu'une croissance économique rapide des pays en développement conditionne la fin de la crise actuelle et l'accès de l'économie internationale à la stabilité.

La première partie du rapport traite de la situation économique internationale actuelle et de ses perspectives à court terme, la seconde partie porte sur le développement historique de l'économie après la deuxième guerre mondiale. La troisième partie est consacrée aux modifications de certains secteurs choisis (entreprises transnationales, produits manufacturés, marchés financiers et transports maritimes internationaux). Un scénario à Iongue échéance à l'usage des pays en développement est présenté dans la quatrième partie.

Selon ce document, les pays en développement se trouvent aujourd'hui confrontés à une situation marquée par la stagnation des revenus, la détérioration des termes de l'échange, l'endettement et le protectionnisme pratiqué par les pays industriels. La continuation d'une période de faible croissance des pays industriels et ses répercussions sur leur structure économique provoqueront une nouvelle aggravation du chômage dans les pays en développement.

Cette croissance économique durablement faible n'est pas acceptable pour les pays en développement; un taux élevé de croissance n'est pas dans leurs possibilités à l'intérieur de l'ordre économique actuel. Pour sortir de ce dilemme, le rapport approuve la possibilité d'un développement endogène des pays en développement. De toutes façons, seules des modifications structurelles dans les pays en développement eux-mêmes pourront conduire au succès. Au chapitre des matières premières, cela signifie qu'il conviendra d'améliorer les termes de l'échange; au chapitre du commerce en général, que les pays en développement devront commercer davantage entre eux, qu'il faudra mieux ouvrir les marchés des pays industriels et substituer les importations par des produits locaux. L'augmentation de l'épargne intérieure, les sources alternatives d'énergie et l'organisation plus rationnelle des ressources économiques doivent aussi être retenues. Enfin, il est nécessaire de renforcer la coopération internationale (38).

\section{L'opinion de la Suisse}

Dans son intervention, le représentant de la Suisse commença par relever que le scénario établi en vue du changement s'inspirait d'une autre optique que celle de la Banque mondiale ou du GATT. Le rapport présente les perspectives économiques des pays industriels sous un jour trop pessimiste et celles des pays en développement sous un jour trop optimiste.

La délégation se déclare persuadée que de plus équitables conditions d'échange pourraient influencer positivement la balance des paiements des pays en développement, fortifier le commerce international et encourager les investis- 
sements. Au demeurant, la Suisse préfère l'ouverture des marchés à leur étranglement. L'intérêt des pays en développement est d'accepter les droits et les obligations d'un système libéral de commerce universel. La Suisse s'étonne de l'importance que le rapport confère à la coopération économique internationale par rapport à la politique nationale de développement. En ce qui concerne le dialogue Nord-Sud, on doit relever une tendance à porter des jugements globaux. La Suisse se demande enfin si un prochain rapport ne devrait pas serrer de plus près les activités de la CNUCED (39).

\section{La 24ème session}

Les membres du conseil de la CNUCED se sont rencontrés du 8 au 19 mars, du 11 au 13 mai et du 28 juin au 2 juillet 1982 pour leur 24ème session fractionnée en trois séances. Ils y ont surtout traité du protectionnisme et des ajustements structurełs et $s^{\prime} y$ sont employés à prendre une décision définitive sur la réalisation de la CNUCED VI.

Cette discussion sur le protectionnisme et les ajustements structurels était une première. Elle résultait d'une décision de la CNUCED V. Dans son premier rapport, le secrétariat de la CNUCED relevait la prolifération des mesures protectionnistes et des accords bilatéraux dans le commerce mondial, ces dispositions ayant pour but de protéger certaines branches de l'industrie, d'empêcher les perturbations du marché et d'équilibrer les balances des paiements. Le protectionnisme exerce une influence pernicieuse sur la croissance économique des pays en développement. C'est pourquoi, dans la mesure où I'on autorise la capacité de concurrence internationale, il est indispensable que les structures se modifient (40).

La délégation suisse s'est également penchée sur cette renaissance du bilatéralisme; elle est d'avis que c'est le principe de la théorie de l'avantage comparatif qui doit inspirer la réforme structurelle. Le représentant de la Suisse combattit au demeurant l'opinion du secrétariat, selon laquelle les barrières douanières élevées par les pays industriels devant les produits des pays en développement seraient deux à trois fois plus hautes que pour les produits en provenance d'autres pays. II rappela les préférences douanières et les hauts tarifs douaniers des pays en développement, ainsi que les particularités du secteur agricole; il estima indispensable qu'on définisse avec précision les perturbations du marché et les obstacles commerciaux non tarifaires (41).

\section{CNUCED VI : un ordre du jour provisoire pour Belgrade}

Le conseil prit la décision d'organiser la CNUCED VI du 6 au 30 juin 1983 à Belgrade/Yougoslavie. Une réunion préparatoire est prévue les 2 et 3 juin, pour les fonctionnaires de haut rang. Le choix s'était à l'origine porté sur Libreville/Gabon, qui dut renoncer à organiser la conférence, la candidature de La Havane/Cuba n'ayant pas été retenue.

Adopté par quatre-vingt-neuf délégations, dont celle de la Suisse, contre les 
voix des Etats-Unis et d'Israël, I'ordre du jour provisoire envisage que la discussion portera sur les thèmes principaux suivants :

- débat général de la CNUCED;

- examen de la crise économique dans l'optique de la politique de développement et à la lumière du "Nouvel ordre économique international" (NOEI); matières premières : état du Programme intégré des matières premières;

- perspectives du commerce international des marchandises et des services, problèmes financiers et monétaires;

- état du nouveau programme d'action substantiel pour les pays les plus pauvres;

- activités de la CNUCED telles que les transferts de technologie, la coopération économique entre les pays en développement, les affaires institutionnelles, etc. (42).

\subsection{Matières premières}

Ces derniers mois, la discussion du Programme intégré des matières premières a été hérissée de difficultés. II a fallu prolonger sensiblement le délai de ratification du Fonds commun pour les produits de base. On ignore encore si le nombre élevé des ratifications indispensables sera atteint.

Au chapitre des accords particuliers sur les matières premières, on a vu l'accord sur le café exercer des effets satisfaisants, l'accord sur le cacao incapable d'empêcher un effondrement des prix, l'accord sur l'étain n'entrer provisoirement en vigueur qu'à une date tardive, alors que l'accord sur le caoutchouc naturel entrait en vigueur à titre définitif. Les négociations sur le thé et les bois tropicaux ont progressé, alors qu'elles piétinaient par ailleurs, par exemple au sujet du coton. Le renouvellement de l'accord sur le blé paraît improbable. La commission des matières premières de la CNUCED presse les négociateurs $d^{\prime}$ intensifier leurs travaux.

La Suisse prend une part active à ces divers pourparlers. Sa participation aux différents accords est en préparation ou déjà un fait accompli (Fonds commun pour les produits de base, étain, caoutchouc naturel, cacaol.

Ces derniers mois, les négociations sur le Programme intégré des matières premières se sont déroulées dans un climat marqué par la forte chute des prix des matières premières. En 1981, la baisse a été d'environ 15\% en moyenne et elle s'est poursuivie en 1982. Aucune matière première n'a été épargnée par cette dégringolade parfois brutale des prix. Les raisons en sont l'offre accrue en provenance des pays en développement et une demande en baisse de la part des pays industriels en proie à la récession.

La politique des matières premières de la CNUCED s'inspire des travaux pré- 
paratoires à l'entrée en vigueur du Fonds commun pour les produits de base, mis sur pied à Genève en juin 1980, et ainsi que de divers autres accords sur les matières premières. Le nombre élevé des ratifications indispensables, nonante, et l'opposition probable des Etats-Unis ne laissent guère subsister l'espoir que le Fonds commun prenne effet dans l'immédiat. Les producteurs de matières premières se demandent s'il ne conviendrait pas de développer la coopération entre les pays en développement plutôt que de miser sur les négociations avec les pays industriels. La question primordiale qui se pose dans le domaine des matières premières est de savoir comment garantir à la fois les gains des producteurs et de plus fortes rentrées en devises.

\section{Etat des diverses négociations}

Pour la majorité des matières premières qui entrent dans le Programme intégré, les négociations se sont poursuivies, soit en vue de renouveler les accords existants, soit pour en préparer de nouveaux. On a provisoirement prolongé d'un an l'accord actuel sur le café, renouvelé les accords existants sur le cacao et l'étain et adopté un nouvel accord sur le caoutchouc naturel. Pour certaines matières premières, aucune disposition n'a pu être prise ou il y a eu trêve de pourparlers (jute, par exemple). On n'a guère avancé d'un seul pas dans le cas des métaux et des minerais. En dehors du Programme intégré des matières premières, on a discuté, à ce jour sans aucun succès, d'un nouvel accord complet sur le blé.

Les clauses économiques de l'accord sur le café de 1976, mises en vigueur pour la première fois en septembre 1980 par le Conseil international du café, ont été prorogées d'un an au cours de l'automne 1981. Les prix du marché sont maintenus à un niveau convenable grâce à un système de quotas d'exportation. Malgré une production mondiale excédentaire, cette réglementation s'est révélée étonnamment efficace à ce jour (43).

Les négociations concernant la conclusion d'un premier accord sur le thé ont enregistré quelques progrès en mai 1982. Les pays producteurs proposent de baser la stabilisation des prix sur une réglementation négociée des quotas d'exportation. On prévoit aussi la création d'un stock servant à parer aux fluctuations des prix à court terme. Les pays consommateurs sont d'avis qu'un stock de ce genre ne jouerait pas son rôle régulateur pour le thé et qu'il risquerait même de faire baisser les prix. Les Etats-Unis se prononcent en outre contre l'introduction de quotas d'exportation. Les négociations se poursuivent (44).

A part diverses séances d'experts, la sixième réunion préparatoire pour les bois tropicaux a eu lieu en juin 1982. On y a pris la décision de convoquer pour 1983 une conférence chargée de conclure un accord international sur les bois tropicaux. Cet accord aura quatre composantes principales :

- développement des programmes de recherches;

- amélioration de l'information concernant les marchés;

- utilisation accrue des bois dans les pays producteurs; 
- reboisement et protection des forêts (sur treize hectares déboisés, un seul est actuellement promis au reboisement).

L'établissement de cet accord jouera un rôle dans le deuxième volet du Fonds commun pour les produits de base (45).

Lors de la troisième réunion préparatoire traitant de la viande (de bœuf), en décembre 1981, on a préconisé l'adoption d'un programme international de recherches, de promotion commerciale et d'autres mesures de valorisation de la viande. Les frais en seraient mis à la charge du deuxième compte du Fonds commun des matières premières et $d$ 'autres institutions financières (46).

Une nouvelle rencontre des pays producteurs et des pays consommateurs de coton s'est déroulée sans succès pour le moment. On y a étudié la possibilité de reprendre les discussions préalables à la signature d'un accord international sur le coton (47).

C'est également sans avoir réalisé l'entente que le Comité du tungstène a mis fin à ses discussions de 1981. Des opinions divergentes subsistent quant à la possibilité et au sens d'un éventuel accord. II y a des années que les tractations concernant ce métal se poursuivent (48).

\section{La séance de la commission des matières premières de la CNUCED}

Réunie en séance du 8 au 12 février 1982, la commission des matières premières de la CNUCED a demandé que les négociations soient accélérées et intensifiées, mettant ainsi à l'épreuve les progrès réalisés au cours des pourparlers sur le Programme intégré des matières premières. A cette réunion, le "Groupe des 77" a fait savoir sa déception de voir les pays consommateurs refuser d'entrer en discussion sur les matières premières, là où ce serait objectivement nécessaire et techniquement possible.

Le porte-parole du "Groupe B" se prononça pour une rapide ratification du Fonds. Selon lui, les pays industriels n'ont rien à voir dans les hésitations de la commission préparatoire. Les pays industriels ont une nette avance sur les pays en développement concernant la signature, la ratification et les préparatifs internes (49).

Le représentant de la Suisse releva certains succès récents au cours des négociations internationales sur les matières premières. A l'échelle nationale, on note que le Parlement helvétique a accepté récemment et sans aucune réserve l'accord international sur le caoutchouc naturel et le projet de création d'un Fonds commun pour les produits de base. Le sixième accord sur l'étain vient d'être transmis aux Chambres fédérales. En juillet 1981, malgré une faible participation internationale, la Suisse s'est également engagée en faveur de l'entrée en vigueur provisoire de l'accord sur le cacao de 1980. 
Discussion autour de la facilité pour les déficits

des recettes $d^{\prime}$ exportation

Le Comité des matières premières de la CNUCED a continué d'étudier la possibilité de créer une facilité pour les déficits des recettes de l'exportation. Une résolution de la CNUCED $V$ demandait au secrétariat de procéder à une étude à ce sujet, en consultation avec le FMI.

La stabilisation des bénéfices provenant des exportations de matières premières des pays en développement est l'un des éléments importants du Programme intégré des matières premières. A I'heure actuelle, le produit des exportations de matières premières est trop bas du fait du niveau des prix imposés aux matières premières fournies par les pays en développement.

Le secrétariat de la CNUCED propose une facilité pour les déficits qui compléterait les actuels systèmes de stabilisation des recettes d'exportation (du FMI et de la CEE) et certaines dispositions figurant au Programme intégré des matières premières. Le "Groupe des 77" espère une dotation financière importante pour cette nouvelle facilité et souhaite la poursuite des études du secrétariat de la CNUCED à ce propos.

Deux opinions s'expriment au sein du "Groupe B" : quelques pays estiment que de nouvelles discussions sur la création des facilités évoquées seront utiles et fructueuses. D'autres, les Etats-Unis en particulier, sont d'avis qu'il incombe au seul $\mathrm{FMI}$ de résoudre ce problème.

La Suisse ne participe ni au système de la CEE (Stabex) ni à celui du FMI pour la stabilisation des recettes d'exportation des pays en développement. Elle étudie actuellement quelle contribution elle pourrait apporter aux négociations sur la facilité par les déficits des recettes d'exportations. Pourtant, dans son message du 9 août 1978 concernant le financement des mesures économiques et commerciales dans le cadre de la coopération au développement, le Conseil fédéral esquissait des lignes d'action possibles (51).

\subsubsection{Fonds commun pour les produits de base}

Le délai de ratification du Fonds commun pour les produits de base, pièce centrale du Programme intégré des matières premières, a été prolongé jus$q u$ 'à fin septembre 1983. I/ faudra donc encore attendre la création du Fonds. La rédaction des dispositions de plein exercice de l'institution avance lentement. La Suisse est au nombre des Etats qui ratifient l'accord.

Pour mettre au point de nouvelles dispositions de plein exercice, la commission préparatoire du Fonds commun des matières premières s'est réunie du 
21 au 25 septembre 1981. Ses deux groupes de travail ont tenu plusieurs séances. Le déroulement languissant des consultations de la commission préparatoire, notamment en ce qui concerne les dispositions d'application opérationnelles et financières du règlement du Fonds, répond au lent processus de signature et de ratification de l'accord, notamment de la part des pays en développement.

\section{Prolongation du délai de ratification}

Aux termes de l'accord conclu, le délai de ratification du Fonds commun pour les produits de base avait été arrêté au 3 mars 1982. A cette date, nonante Etats au moins auraient dû avoir signé et ratifié l'accord. Ils auraient aussi dû réunir entre eux les deux tiers au minimum des contributions directes (460 millions de dollars EU). On aurait dû avoir l'assurance que la moitié au moins des contributions bénévoles serait versée en vue de la réalisation du deuxième compte ( 280 millions de dollars EU). A fin mars 1982, les conditions posées à l'entrée en vigueur du Fonds des matières premières étaient cependant loin d'être remplies. Quatre-vingt-deux Etats seulement avaient contresigné le document et vingt-cinq Etats en tout et pour tout I'avaient ratifié. Afin de sauvegarder le caractère universel du Fonds commun, on ne saurait renoncer à un nombre relativement élevé de pays membres.

En juin 1982, lors d'une conférence réunissant les vingt-cinq Etats qui ont ratifié dans les délais le Fonds commun pour les produits de base, on décida de reporter au 30 septembre 1983 le délai de ratification. Début juillet 1982 : quatre-vingt-six Etats avaient signé l'accord et trente-deux l'avaient ratifié. Les cosignataires représentent $24 \%$ des capitaux nécessaires.

\section{Les parlementaires suisses se prononcent en faveur de l'accord}

C'est en avril 1980 déjà que la Suisse annonçait son intention de faire partie du Fonds et de lui fournir une contribution bénévole. Après publication du message du 25 février 1981 du Conseil fédéral sur les mesures commerciales et les mesures relatives aux produits de base dans le cadre de la coopération au développement, la Suisse a signé l'accord sur la création du Fonds commun pour les produits de base le 30 mars 1981. Suivant les recommandations du message sus-mentionné, le Parlement a donné son assentiment à l'unanimité, le 30 septembre 1981, à la participation de la Suisse à l'accord dont nous parlons, ouvrant ainsi au Conseil fédéral les portes de la ratification. Le délai de référendum s'étant écoulé sans être utilisé, le Conseil fédéral a décidé de la ratification en mai 1982, cet acte devant être accompli au cours de l'été.

La contribution de la Suisse au premier compte s'élève à mi-1982 à environ 8,3 millions de francs et sa contributions bénévole au deuxième compte à 11,5 millions de francs. Ces deux contributions sont prises sur les fondscadres pour le financement des mesures de politique économique et commerciale inspirée par la coopération au développement. 
Comme l'indique le Conseil fédéral dans son message sur les mesures à prendre concernant sa politique commerciale et de mesures relatives aux produits de base, une participation financière au Fonds commun pour les produits de base est l'un des points marquants du débat sur les matières premières.

La plupart des pays en développement continuent de vivre dans la dépendance des exportations de quelques matières premières. De fortes et brutales oscillations des prix ont des conséquences désagréables pour pays en développement et pays industriels. Dans les pays en développement, la planification de l'essor économique se trouve modifiée, car la baisse des prix rend indispensables des crédits qui aggravent l'endettement. Dans les pays industriels, difficultés d'achat et de stockage pour les industries de transformation. Pays décidément pauvre en matières premières, la Suisse soutient depuis longtemps les efforts de la communauté internationale en vue de stabiliser le marché mondial de ces produits. Les initiatives prises en commun par les pays en développement et les pays industriels rendent moins vraisemblable la formation de cartels de matières premières par les pays en développement (53).

\subsubsection{Sixième accord international sur l'étain}

Ce n'est que tardivement et provisoirement que l'accord est entré en vigueur. Les grands pays consommateurs bloquent une entrée en vigueur définitive. Malgré une faible participation des pays consommateurs, l'accord a été mis provisoirement en vigueur le 1er juillet 1982; trois pays producteurs ont conclu une entente entre producteurs. Première participation de la Suisse à l'accord.

En juin 1981, la conférence réunie à Genève par les Nations Unies a couronné ses travaux en concluant le Gème accord sur l'étain (entrée en vigueur définitive ou provisoire prévue pour le 1er juillet 1982); les Etats intéressés ont pu signer le document.

Après l'échéance du délai de signature fixé à fin avril, il était à prévoir que, vu la faible participation des pays consommateurs, les conditions d'une entrée en vigueur automatique ne pourraient guère être remplies. Pour y parvenir, il aurait fallu que $65 \%$ au moins de la part prise au marché mondial fussent représentés, tant du côté des producteurs que du côté des consommateurs. Dix-neuf pays seulement avaient signé l'accord, représentant 79\% de la production mondiale, mais seulement $48 \%$ de la consommation mondiale. Comme ils I'ont déjà fait savoir lors de la conférence sur l'étain de 1981, les Etats-Unis, qui sont le premier consommateur mondial $(27 \%)$, ne 
sont plus partie prenante au 6ème accord et bloquent ainsi son entrée en vigueur définitive. Autre consommateur important avec sa part au marché d'environ $10 \%$, I'Union soviétique ne souscrit plus au nouvel accord, de sorte que la barre des $65 \%$ est inaccessible côté consommateurs.

Les pays fournisseurs n'avaient réuni que $79 \%$ de la production totale, soit un peu moins que les $80 \%$ exigés pour une entrée en vigueur définitive de I'accord. Accident de parcours sans conséquence, puisque les conditions de I'entrée en vigueur provisoire étaient remplies. La Bolivie manque encore à l'appel $(15,6 \%)$, ce qui n'étonne personne après ce qu'on a entendu dire à I'issue de la conférence de I'ONU sur l'étain.

Fin juin 1982, les dix-neuf Etats qui avaient déjà ratifié l'accord ou qui s'étaient engagés à en faire usage, décidaient de le mettre provisoirement en vigueur au 1er juillet 1982 malgré la faible participation côté consommateurs.

La marché de l'étain, où la ligne de démarcation entre producteurs des pays en développement et consommateurs des pays industriels est assez nette, connaît depuis quelques années une offre excédentaire. Vers la fin de l'année, un puissant groupe financier - opérant très vraisemblablement à partir de la Malaisie - a tenté de s'y assurer le contrôle de l'offre. Cette offensive a échoué, mais le Conseil de l'étain a dû effectuer des achats de soutien. En outre et depuis longtemps, les Etats-Unis écoulent par petites quantités une part de leurs réserves stratégiques d'étain, autre forme de pression sur les prix à longue échéance. Les pays producteurs n'épargnent pas leurs critiques aux Etats-Unis à ce sujet.

Au cours de sa séance de mars, le Conseil de l'étain a évoqué la possibilité de placer les exportations sous contrôle pour soutenir les prix du métal; il a aussi discuté de la mobilisation de nouvelles ressources financières afin d'accroître les stocks compensatoires. Il a pris des mesures draconiennes pour régler les exportations et a adopté des crédits élevés (54).

Dans le camp des producteurs, on a noté de la part de la Malaisie des efforts pour édifier un cartel de l'étain (TINPEC). D'abord hésitantes, I'Indonésie et la Thaïlande s'y sont jointes. Finalement, c'est-à-dire au moment où I'entrée en vigueur du nouvel accord sur l'étain ne faisait plus de doute, cette entente entre producteurs fut présentée non pas comme un cartel des prix, mais comme l'instrument d'une meilleure harmonisation des intérêts de ses membres. II est possible qu'il joue un rôle important si le 6ème accord international sur l'étain devait rester inopérant (55).

\section{Le Conseil fédéral recommande l'adhésion de la Suisse}

La Suisse n'a jamais été membre de l'accord sur l'étain. Dans son message du 20 janvier 1982 sur le 6ème accord international sur l'étain, le Conseil fédéral demande au Parlement de se prononcer pour l'adhésion. Dans sa session d'été (23 juin 1982), le Conseil national s'est déjà prononcé favora- 
blement à l'unanimité.

Le Conseil fédéral estime que l'accord international sur l'étain a fait ses preuves. II a contribué à stabiliser le marché et les prix de l'étain. Le 6ème accord jouera certainement un rôle important dans l'instauration du Fonds commun pour les produits de base. Même utilisé en petites quantités, l'étain est une matière première importante, sujette à de multiples usages, pour beaucoup d'entreprises industrielles suisses. En 1981, la Suisse a importé pour 25 millions de francs d'étain brut (les réexportations d'étain brut représentant une valeur de 3,7 millions de francs durant la même année); la Suisse a également importé en 1981 pour 17 millions de francs de soudure d'étain ou d'autres dérivés.

La contribution financière de la Suisse à l'accord est évaluée selon la part prise à la consommation mondiale, qui était de 0,45\% en 1978-1980. En conséquence, les engagements financiers pour le "stock compensatoire normal" s'élèvent approximativement à 2,8 millions de francs. Cette contribution pourrait être complétée par une éventuelle garantie de 2 millions de francs au maximum pour le stock complémentaire. Cet apport est prélevé sur le crédit-cadre pour les mesures de politique économique et commerciale à prendre dans le cadre de la coopération technique. S'y ajoutent chaque année environ 20.000 francs de contribution aux frais de gestion du Conseil de l'étain.

La communauté de travail Swissaid (i3m)/Action de Carême/Pain pour le prochain/Helvetas accueille favorablement I'adhésion de la Suisse. II s'agit d'un appui donné précisément dans une phase de négociations difficiles, au Programme intégré des matières premières (57).

\subsubsection{Accord international sur le caoutchouc naturel}

L'accord international sur le caoutchouc naturel est entré définitivement en vigueur; les principaux pays producteurs et consommateurs en font partie. La Suisse a souscrit à l'accord à mi-juillet 1982.

L'accord international sur le caoutchouc naturel est entré définitivement en vigueur le 15 avril 1982. II avait été conclu le 6 octobre déjà et était entré provisoirement en vigueur le 23 octobre 1980.

Pour l'entrée en vigueur définitive, les Etats membres devaient réunir au moins $80 \%$ de la production et de la consommation mondiales. Ils finirent même par représenter $91,4 \%$ de la production et $84,8 \%$ de la consommation. Les pays membres sont actuellement au nombre de trente, dont six producteurs, et parmi eux les trois plus grands, la Malaisie, I'Indonésie et la Thaï- 
lande, les pays consommateurs étant vingt-quatre (dont les cinq grands : Etats-Unis, CEE, Japon, Chine et Union soviétique).

Intervenue dans les délais, l'entrée en vigueur définitive ne fut possible qu'au moment où quelques pays( dont la Thaïlande et la $C E E$ ) déposèrent les actes nécessaires à New York peu avant l'expiration du délai de ratification qui avait été prolongé de six mois. Vraisemblablement, l'accord restera en vigueur jusqu'en octobre 1985.

L'accord vise à stabiliser les prix du caoutchouc gràce à la constitution d'un stock compensatoire. Ce stock sera financé à parts égales par les pays producteurs et les pays consommateurs. Les prix devront être stabilisés à un prix de référence de $210 \mathrm{cts} / \mathrm{lbs}$ (Malaisie/Singapour), l'écart vers le haut et vers le bas étant fixé à $20 \%$. Dès la mise en œuvre du Fonds commun pour les produits de base, l'accord y sera intégré. Vu la baisse des prix et la légère diminution de la consommation en 1981, les pays producteurs ont demandé que soient renforcées les possibilités d'intervention pour stabiliser les prix (58).

La Suisse participe à cet accord depuis peu de temps. Le 9 octobre 1981, l'assemblée fédérale avait déjà approuvé I'accord international sur le caoutchouc naturel et autorisé le Conseil fédéral à y adhérer. Le droit au référendum n'ayant pas été invoqué, le Conseil décida de son adhésion le 26 mai et agit dans ce sens à fin juillet.

Selon le Conseil fédéral, l'économie suisse n'accorde plus la même importance qu'avant au caoutchouc naturel, mais reste intéressée à la stabilisation du marché mondial de cette matière première. L'accord sur le caoutchouc naturel est le premier et jusqu'ici le seul exemple d'une entente complète entre producteurs et consommateurs dans le cadre du Programme intégré des matières premières.

\subsubsection{Accord international sur le cacao}

Entré provisoirement en vigueur, l'accord international sur le cacao n'est pas parvenu à stabiliser les prix au niveau prévu. Une offre trop abondante et l'absence des deux principaux pays producteurs et consommateurs accroissent les difficultés de stabilisation du marché.

A l'occasion de l'entrée en vigueur provisoire, le 1er août 1981, de l'accord international sur le cacao, le Conseil fédéral publia le 21 septembre 1981 l'ordonnance qui permet à la Suisse d'en réaliser les termes. Cette ordonnance a pris effet au 1er octobre 1981.

En Suisse, selon les prescriptions du Conseil international du cacao, c'est I'Office fiduciaire des imıportateurs suisses de denrées alimentaires (OFIDA) 
qui se charge des contrôles prévus. Les importateurs suisses doivent maintenant acquitter une taxe de 2,20 $\$$ (très probablement $4,40 \$$ à partir du 1 er octobre 1982) par $100 \mathrm{~kg}$ de fèves de cacao importées et en provenance de pays ne participant pas à l'accord. Cette taxe et des contributions analogues prélevées par les pays exportateurs sur leurs ventes de cacao aident à financer la constitution d'un stock compensatoire de cacao (60).

Lors de l'entrée en vigueur de l'accord, le prix du cacao était en dessous du prix d'intervention convenu, de sorte que le stock compensatoire a dû procéder aussitôt à des achats de soutien. Les prix pratiqués sur le marché auraient ainsi dû se stabiliser au niveau de $110 \mathrm{cts} / \mathrm{lbs}$, minimum prévu dans I'accord.

D'un volume d'environ 100.000 tonnes de fèves de cacao, les achats de soutien n'ont pas suffi à faire remonter les prix au niveau souhaitable. Selon les dispositions de l'accord, la limite inférieure des prix d'intervention a été ramenée de 110 à 106 cts en mars 1982. Dès lors, les achats de soutien se sont arrêtés par manque de ressources financières, de sorte que les prix du marché sont de nouveau tombés, comme c'était le cas avant l'entrée en vigueur de l'accord, à $70-75 \mathrm{cts} / \mathrm{lbs}$.

Au Conseil du cacao, les négociations sur une solution à échéance aussi lointaine que possible se poursuivent malgré des échecs répétés. On discute de I'augmentation des taxes ou d'une nouvelle fixation des prix d'intervention. Un prêt de 75 millions de $\$$ pour financer de nouveaux achats de soutien a été signé avec un groupe bancaire brésilien. Aucune entente entre pays producteurs et pays consommateurs n'a cependant pu être obtenue concernant l'utilisation de ces moyens financiers.

L'offre structurelle excédentaire et l'accroissement des stocks ne laissent guère entrevoir à court terme un relèvement des prix du marché. La Côted'Ivoire, premier pays producteur et exportateur, ainsi que les Etats-Unis, principal pays consommateur, restent au demeurant en dehors de l'accord (61).

\subsubsection{Accord international sur le blé}

Les accords sur le commerce du blé et l'aide alimentaire ont été prorogés de deux nouvelles années en date du 1er juillet 1981. L'opposition manifestée par les grands pays exportateurs continue d'empêcher que l'entente se fasse sur un nouvel accord global.

Cet accord comporte deux instruments de droit : le texte de 1971 portant sur le commerce du blé et celui de 1980 concernant l'aide alimentaire. La validité de ces deux documents a été prorogée de deux ans au 1er juillet 1981, 
pour la sixième fois quant au premier, pour la première fois quant au second. La discussion a porté une fois encore sur la conclusion d'un nouvel accord global sur le blé. Au cours des douze mois écoulés, aucune entente n'a pu se réaliser entre exportateurs et importateurs, surtout pas sur le problème du financemenie-et du contrôle des stocks. On propose de constituer des stocks nationaux dans le cadre d'une coordination internationale. De l'avis du Conseil international du blé, cette mesure engendrerait une plus grande stabilité du marché et garantirait une meilleure sécurité alimentaire. La majorité des pays membres, dont la Suisse, ont approuvé cette suggestion, mais les EtatsUnis, le Canada et I'Australie, les trois plus grands exportateurs de blé, l'ont refusée.

Les douze signataires de l'accord sur l'aide alimentaire ont pris l'engagement de fournir aux pays en développement un minimum annuel de 7,6 millions de $t$, de céréales. Des contributions financières pour l'achat de céréales et de produits céréaliers aux prix du marché peuvent remplacer les dons en nature. Pays importateur de céréales, la Suisse fournit une contribution de solidarité de 27.000 t de céréales par an. Cette aide s'effectue sous la forme de farine fabriquée en Suisse ou de céréales et de farine achetées à l'étranger. Pour I'année courante, 16 millions de francs étaient disponibles à cet effet (62).

L'accord de 1971 sur le commerce du blé est un contrat-cadre peu contraignant qui encourage surtout les échanges d'informations entre les grands acheteurs et vendeurs de blé. Ce texte ne comporte pas de dispositions d'ordre économique, rien qui touche aux prix ou aux quantités (63). L'entente n'ayant pu se faire sur un nouvel accord global, il est probable que ce contratcadre soit de nouveau prolongé de trois dans un proche avenir et que sa validité soit étendue à fin juin 1986.

En 1981/82, on s'attend à une nouvelle moisson record d'environ 460 millions de t., dont le commerce international devrait écouler 100 millions de $t$. L'an passé, on avait enregistré une production en hausse des exportateurs traditionnels, une hausse des prix et une baisse des stocks (64).

\subsection{Produits manufacturés}

\subsubsection{Préférences douanières}

Le comité spécial de la CNUCED a vérifié le système des préférences douanières. Le secrétariat de la CNUCED et le "Groupe des 77 " se sont prononcés en faveur d'une généralisation, le "Groupe B" a rappelé la prolongation des accords et le bas niveau de leur utilisation. La Suisse a également fait savoir qu'elle était en faveur d'une prolongation et d'une extension des préférences 
comme de la possibilité d'y introduire une clause de gradation.

Le comité spécial des préférences de la CNUCED a tenu sa 11ème séance du 3 au 11 mai. Au terme des dix premières années d'existence des préférences douanières, le comité spécial désirait passer au peigne fin leur usage et leur extension.

Le secrétariat de la CNUCED a fait savoir qu'une différenciation des préférences douanières octroyées aux pays en développement en raison de leur développement économique n'était pas compatible avec les principes fondamentaux du schéma des préférences douanières. On peut utiliser la clause de différenciation à titre de protection, mais on ne saurait en faire un élément durable du SGP (système général de préférences). Les pays industriels qui recourent à cette différenciation le font, faute d'avoir su adapter à temps leurs structures à l'accroissement des importations, pour parer aux difficultés économiques de leurs industries nationales. La clause de différenciation devrait permettre d'exclure des préférences douanières certains produits en provenance de pays en développement fortement concurrentiels. C'est là un moyen pour les pays en développement de tirer un parti plus avantageux du SGP. Or, c'est loin d'être le cas, selon l'avis de la CNUCED. Ce mécanisme contribuerait plutôt à réduire le nombre des pays en développement favorisés.

Le "Groupe des 77" a réclamé entre autres dans une résolution I'amélioration du statut juridique du schéma des préférences douanières, sa généralisation et la levée des limitations, et qu'on procède à une expertise multilatérale sur les limitations en vigueur. Cette résolution sera examinée au cours de la prochaine session.

Le "Groupe B" a fait remarquer que les pays industriels avaient prolongé de dix ans la validité des préférences douanières. Le volume du commerce avec les pays de I'OCDE qui est soumis au régime des préférences douanières a certes atteint 25 millions de dollars EU en 1980, mais les pays en développement n'en retirent pas encore tout le bénéfice possible. En outre, le "Groupe B" s'est opposé aux exigences du "Groupe des 77 " concernant la multilatéralisation, I'unification et l'extension des préférences douanières (65).

La Suisse a fait savoir qu'elle avait également prolongé de dix ans la validité de ses préférences douanières, en incluant toutefois une possibilité de gradation. Ce faisant, elle tient compte du dynamisme du développement économique et des échanges croissants avec les pays en développements. Les plus avancés de ces pays devraient peu à peu assumer leurs droits et leurs obligations dans le système des échanges universels. S'ils désirent continuer à bénéficier de préférences douanières, les pays doivent, à longue échéance, ouvrir leurs marchés.

A l'heure actuelle, la Suisse n'a encore jamais eu recours à la clause de sauvegarde. Le degré d'utilisation des préférences douanières (1981:36\%) pour- 
rait être amélioré. La Suisse a en outre annoncé qu'elle étendrait ses préférences douanières aux trente et un pays les plus pauvres. Ceux-ci bénéficient de l'exemption des droits de douane pour presque tous leurs produits industriels, aussi pour les textiles, I'habillement et les chaussures, ainsi que pour quelques produits agricoles. Enfin, des préférences douanières ont été accordées à de nouveaux pays et à d'autres produits. Une limitation des préférences douanières ne saurait cependant pas être mesurée uniquement à l'aune de la capacité économique des pays en développement, c'est-à-dire sur la base du revenu par tête d'habitant (66).

\subsubsection{Facilité de la CNUCED de garantie du crédit à l'exportation}

Un groupe d'experts a recommandé l'instauration d'une telle commodité. L'entente a pu se faire de manière informelle sur les détails techniques, mais on ignore si le projet pourra etre accepté au niveau politique. L'incertitude est de mise vu le faible intérêt démontré par maint pays en développement et les doutes sérieux que nourrissent de grands pays industriels. Cette facilité de garantie viendrait en aide aux pays en développement qui se lancent dans le financement de leurs exportations de biens d'investissements et de biens de consommation durables. La Suisse s'intéresse en principe à ce projet.

Du 11 au 22 janvier 1982, un groupe d'experts de la CNUCED a siégé à Genève pour étudier la création d'une facilité de garantie du crédit à l'exportation. La convocation de ce groupe d'experts avait été décidée, selon une résolution de la CNUCED, à la 9ème session de la CIFT (Commission des invisibles et du financement lié au commerce). Le secrétariat fut chargé de mener les consultations et de proposer un projet.

La facilité prévue garantirait les crédits à l'exportation à moyen et à long terme, qui sont consentis par les pays en développement. Pour l'essentiel, il s'agirait de couvrir avec des capitaux de toute confiance le financement préliminaire des investissements requis par les exportations de biens de consommation durables. Le but de l'opération est de contribuer à atténuer le déficit de la balance des paiements des pays en développement. L'instauration de la facilité prévue donne aux pays en développement la possibilité d'accorder davantage de crédits à l'exportation. Elle améliore ainsi leurs positions dans la concurrence internationale face aux pays industriels qui financent également les exportations de ce type.

Au sein du "Groupe des 77", on estime que cette facilité ne présente guère d'intérêt pour les pays latino-américains et ceux d'Afrique. Dans un avenir prévisible, elle n'aidera qu'environ vingt à trente pays en développement qui 
commencent tout juste à exporter des biens d'investissement ou des biens de consommation durables. Les autres pays en développement en tireront parti tout au plus en qualité d'importateurs; on peut y voir aussi un encouragement aux échanges commerciaux entre les pays en développement eux-mêmes. Au sein du "Groupe B", I'opposition fondamentale des pays anglo-saxons à I'instauration de la facilité proposée se maintient, alors que divers petits pays d'Europe et de la CEE désirent étudier plus à fond le mécanisme proposé avant de franchir le pas.

De façon informelle, les experts du groupe sont parvenus à se mettre d'accord sur certains aspects opérationnels de la facilité et à préparer toute une série de questions techniques à élaborer par le secrétariat de la CNUCED. Les consultations du groupe d'experts restent libres de tout engagement, d'autres séances sont attendues. L'instauration de la facilité prévue semble techniquement possible, mais on se demande si elle sera acceptée au niveau politique. La prochaine session de la CIFT décidera de la suite des opérations (67).

A certaines conditions, la Suisse est tout à fait disposée à traiter de la dite facilité de garantie du crédit à l'exportation. II y aurait là de quoi encourager les exportations de biens d'investissement et de biens de consommation durables en provenance des pays en développement (68).

\subsubsection{Pratiques commerciales restrictives}

L'ONU surveille l'application du code pour le contrôle des pratiques commerciales restrictives. Lors de la révision de la loi suisse sur les cartels, l'application de ce code semble peu probable.

En 1980, les Nations Unies ont accepté sous forme de recommandation le code sur les pratiques commerciales restrictives. La Suisse a participé à l'élaboration de ce code et elle s'est associée aux termes de la recommandation. Prévue par le code, la première session du groupe de travail intergouvernemental pour le contrôle des pratiques commerciales restrictives s'est tenue à Genève, du 2 au 11 novembre 1981. La fonction essentielle du groupe de travail est de veiller à l'application du code de contrôle des pratiques commerciales restrictives.

Les participants à cette première séance ont été informés de la publication et de I'application du code.dans les divers Etats et de l'élaboration des lois nationales sur les pratiques commerciales restrictives. Le groupe de travail constate qu'on continue à recourir à des pratiques commerciales restrictives dans le commerce international et il invite les Etats à contrôler ces agissements. Il attend du secrétariat de la CNUCED qu'il propose des modèles de 
lois sur les pratiques commerciales restrictives. Pour le reste, le secrétariat est chargé de réaliser des études sur certaines pratiques commerciales restrictives. On lui demande aussi de réaliser des programmes de consultations et de formation (69).

A l'instar des gouvernements d'autres pays industriels, le Conseil fédéral a publié le code en attirant l'attention des entreprises suisses sur son existence. Les dispositions figurant dans la révision de la loi sur les cartels et que le Conseil fédéral propose pour la conclusion d'accords internationaux ne peuvent être utilisées en rapport avec le code, dit le message, car le code n'est pas en soi un accord international; il ne représente qu'une recommandation (70).

La communauté de travail Swissaid/Action de Carême/Pain pour le prochain/ Helvetas, s'inspirant de l'attitude positive de la Suisse face au code, a proposé qu'on tienne compte des impératifs de la politique de développement lors de la révision de la loi sur les cartels. II y a lieu de stigmatiser les pratiques commerciales des cartels internationaux domiciliés en Suisse, qui portent préjudice aux intérêts économiques des pays en développement. Ce faisant, on soulignerait la cohérence de la politique suisse du commerce extérieur et du développement (71).

\section{GATT}

// faut relever dans les travaux du GATT en 1981 et 1982 la conclusion des négociations sur la prolongation de l'accord concernant les multifibres, la préparation d'une réunion ministérielle en novembre 1982 et l'examen de la politique agricole de la Suisse en ce qui concerne la Confédération.

La dernière réunion ministérielle du GATT avait eu lieu en 1973 à Tokyo. La prochaine déterminera le rôle du GATT dans les annés 80 après les modifications intervenues dans le commerce mondial ces dernières années, modifications provoquées notamment par la progression de divers pays en développement. II s'avère indispensable de passer en revue l'actuel système de commerce multilatéral, afin de le renforcer et de le compléter si nécessaire.

Les discussions vont donc se concentrer sur cinq points primordiaux :

- consolidation des résultats obtenus à Tokyo;

- difficultés des échanges internationaux (par exemple définition des clauses de sauvegarde);

- intégration des pays en développement dans le commerce mondial;

- coopération dans le domaine de l'agriculture;

- tâches du GATT au cours des années 80 (72). 
En tant que pays directement associé aux travaux préparatoires, la Suisse est concernée par les points suivants de la réunion ministérielle :

- meilleur respect, renforcement des règlements du GATT et leur adaptation aux réalités des années 80 ;

- réglementation des clauses de sauvegarde;

- intégration accrue des pays en développement au système du commerce mondial;

- amélioration des procédures permettant de régler les différends (73).

\section{Le troisième accord sur les multifibres (AMF 3)}

Le 22 décembre 1981 se sont terminées à Genève les négociations sur la prolongation de I'AMF qui reste en vigueur jusqu'au 31 juillet 1986. C'est dans le cadre du GATT qu'avait été conclu le premier AMF qui fut prolongé au début de 1978 jusqu'à la fin 1981 et qui vient de l'être une deuxième fois. Des quarante et un pays qui avaient souscrit à cet accord, trente-trois avaient signé le protocole de prorogation à fin juin 1982, y compris la Suisse (sous réserve de ratification par le Parlement).

L'accord détermine le cadre multilatéral des négociations bilatérales engagées entre pays importateurs et pays exportateurs. En autorisant un protectionnisme limité, on souhaite favoriser une progression ordonnée du commerce international des textiles. A l'exception des matières premières, l'accord porte sur toutes les phases de fabrication de l'industrie des textiles et du vêtement. Les pays exportateurs se voient garantir un taux de croissance minimum de leurs ventes de textiles. Les pays importateurs obtiennent certaines mesures de protection de leurs industries et de leurs marchés.

L'accord couvre à peu près $80 \%$ du commerce mondial des textiles pour une valeur qu'on estime à environ 80 milliards de $\$$. Si I'on jette un coup d'œil au marché mondial des textiles, on constate que les pays industriels y sont les plus actifs avec $73 \%$ des exportations de textiles et $50 \%$ des exportations de vêtements. Les pays en développement s'assurent $20 \%$ des exportations de textiles et $37 \%$ des exportations de vêtements. La part des pays en développement aux importations de textiles des pays industriels (textiles et habillement) est d'environ $28 \%$. Face aux pays en développement, les pays industriels enregistrent une balance commerciale excédentaire dans le commerce des textiles (1980 : plus 2,29 milliards de $\$$ ) et une balance déficitaire dans le commerce de l'habillement (1980: moins 11,39 milliards de \$).

L'AMF 3 ne modifie en rien le texte de l'accord précédent, mais le protocole de prorogation présente quelques dispositions finales fort efficaces, qui freineront les relations commerciales davantage qu'avant. Les innovations suivantes sont à signaler :

- le mécanisme "anti-surge" permet dans certaines conditions aux pays importateurs de se défendre contre l'importation excessive de produits menaçant leur économie. Les exportateurs recevront des compensations équitables; 
- lors de perturbations du marché dans les pays importateurs, les taux de croissance pourront être abaissés au-dessous des $6 \%$ prévus.

Ouvertes au cours du printemps, ces difficiles négociations ont été caractérisées par la volonté commune des pays en développement d'obtenir une plus large ouverture des marchés des pays industriels. Les pays scandinaves et de la CEE ont au contraire pris position pour un protectionnisme accru. Les Etats-Unis ont joué un important rôle d'arbitre. On pense que des mesures inspirées par un protectionnisme accru vont être proposées au cours des négociations bilatérales par la CEE (74).

La Suisse fit savoir que la prorogation de l'accord éliminait certaines incertitudes du marché international des textiles et qu'elle tenait pour équilibré le résultat des négociations. Au cours des pourparlers, la Suisse rappela qu'à l'origine l'accord devait parvenir à créer un instrument susceptible de régulariser le marché du textile. Un marché libre devrait être possible dans l'avenir. L'AMF ne saurait se cantonner dans un protectionnisme constant. Les marchés des pays industriels et des pays en développement devraient s'ouvrir peu à peu. Dans le cadre de I'AMF, les Etats devraient entrer dans une concurrence loyale, c'est à quoi doit servir la protection des biens immatériels, par exemple des marques et des désignations d'origine. A ce jour, la Suisse n'a pas fait usage des mesures protectionnistes offertes par l'accord (75).

\section{Examen de la politique d'importations agricoles de la Suisse}

En 1966, lors de son entrée définitive dans le GATT, la Suisse a obtenu un statut spécial qui lui permettait, cas unique, d'édicter lors de la promulgation de sa loi sur l'agriculture des restrictions aux importations. Elle devait s'engager à présenter chaque année un rapport au Conseil du GATT et à se soumettre en principe tous les trois ans à un examen effectué par un groupe de travail.

En vertu d'une demande du Chili, appuyée par les grands pays exportateurs de produits agricoles que sont l'Argentine, I'Australie, le Canada et les EtatsUnis, le Conseil du GATT décida en décembre 1981, au cours de sa 37ème séance, de faire examiner par un comité de travail la politique agricole de la Suisse. Lors de cette expertise on examine en premier lieu la politique suisse d'importations agricoles. II fut démontré que l'approvisionnement de la Suisse dépend largement des importations agricoles. Seulement 55\% des besoins caloriques humains sont couverts par le marché intérieur. Les importations agricoles en provenance des pays en développement représentent environ $20 \%$ du total. D'autre part, une analyse détaillée de certaines positions commerciales a été faite, en particulier en ce qui concerne la viande et le système des contingentements, en trois phases. La Suisse tint à préciser que I'accés au marché était garanti et que I'on ne pouvait pas parler d'un protectionnisme accru.

Dans sa séance de mai, le Conseil du GATT prit connaissance du rapport de 
ce comité de travail. L'examen subi par la Suisse s'est terminé de manière satisfaisante, mais les restrictions à l'importation feront l'objet d'un contrôle suivi (76).

Au 1er janvier 1982, la Suisse a fait entrer en vigueur la troisième des huit étapes de suppression des droits de douane qui ont été convenues lors des négociations commerciales multilatérales de la réunion du GATT de 1979, à Tokyo. "Le taux des droits de douane perçus sur les positions figurant dans les premières étapes se trouve ainsi abaissé d'un huitième par rapport aux diminutions convenues. Pour les positions (notamment dans le domaine des textiles, de l'acier et de la céramique) où les droits de douane ne doivent être abaissés qu'au début de 1982, la diminution des taux représente un sixième de la réduction convenue" (77).

\section{FONDS MONETAIRE INTERNATIONAL (FMI) ET BANQUE MONDIALE}

Deux événements importants à retenir : le Conseil fédéral envisage favorablement l'entrée de la Suisse au FMI et à la Banque mondiale; I'AID (Association internationale de développement) éprouve des difficultés financières.

L'entrée éventuelle de la Suisse au FMI est restée en suspens en 1981, mais les études préliminaires ont marché bon train. Un groupe de travail interdépartemental a déposé fin 1981 son rapport sur l'entrée de la Suisse et le Conseil fédéral s'est prononcé de manière positive en août 1982. Les facteurs qui militent le plus en faveur d'une entrée au FMI sont le maintien d'un ordre monétaire stable avec liberté des échanges commerciaux et des paiements, ainsi que la contribution du FMI au financement des balances de revenus déficitaires et cela dans un climat de plus grande interdépendance des politiques monétaires (78).

En faveur d'une entrée à la Banque mondiale, on relèvera I'harmonie des lignes essentielles de la politique de développement suivie par la Banque mondiale et la Suisse, la possibilité de se faire mieux entendre dans le dialogue Nord-Sud et d'exercer un contrôle plus suivi des contributions mises à la disposition de I'AID. Entrer à la Banque mondiale, c'est aussi compléter I'appareil d'une politique de développement et exprimer plus complètement la solidarité internationale de la Suisse. C'est encore ouvrir à l'économie un accès sûr aux émissions de la Banque mondiale.

Lors de la 36ème conférence annuelle du FMI et de la Banque mondiale à Washington, du 29 septembre au 2 octobre 1981, la Suisse fut le seul pays admis en tant qu'observateur. Lors de la session de printemps du comité 
commun de développement, les 13 et 14 mai 1982 à Helsinki, la Suisse était également représentée avec un statut d'observateur. On y discuta des présentes difficultés de financement de l'AID. L'impasse a été provoquée par le refus américain de verser les contributions des Etats-Unis aux tranches prévues. La sixième étape de renouvellement des fonds de I'AID est ainsi remise en question, comme le financement de nouveaux projets pour les années 1980-83.

La France proposa de créer un fonds spécial "AID 6 bis". Cette idée a eu I'appui de divers Etats européens et du Canada. Les pays donateurs réagissent ainsi pour parer au retard des versements des Etats-Unis. "AID 6 bis"' agirait de concert avec I'AID. La création de ce fonds nouveau aurait des répercussions sur l'économie suisse. A ce jour, celle-ci a pu participer aux émissions servant à financer les projets de l'AID. A l'avenir, les projets d" "AID 6 bis" ne seraient financés que par des émissions émises dans les pays participant au fonds. La Suisse n'a pas pris position concernant la proposition française (79).

II convient de signaler les relations financières entre le $\mathrm{FMI}$, la Banque mondiale et la Suisse. Le crédit ouvert par la Banque nationale suisse (BNS) à la facilité Witteveen du FMI pour un montant de 650 millions de DTS fut débité en 1981 de 197,4 millions de DTS. A fin 1981, 322,3 millions de DTS restaient disponibles. Pour financer les octrois de crédits de la part du FMI, la Banque nationale accorda en 1981 une nouvelle tranche de 150 millions de DTS. Les crédits récupérables que la Banque nationale a octroyés au FMI pour des achats de pétrole, crédits partiellement garantis par la Confédération, sont revenus en 1981 à 74,7 millions de DTS par suite de remboursements (80).

Au cours de l'année 1981-82 la place financière suisse a été, en importance, le deuxième fournisseur de capitaux de la Banque mondiale. Les différentes émissions sont résumées dans le tableau ci-dessous.

\section{EMPRUNTS BANQUE MONDIALE} en millions FS - Exercice 1981-82

Souscriptions publiques

Obligations $8 \%$ à 10 ans, échéance 1991

100,0

Obligations 7,25\% à 10 ans, échéance 1992

100,0

Obligations 7,25\% à 10 ans, échéance 1992

100,0

Placements auprès de banques centrales

Obligations 7,625\%, échéance 1988-90

400,0

Obligations 7,50\%, échéance 1988

100,0

Bons $5,875 \%$ à 2 ans, échéance 1984

50,0

Obligations 7,25\%, échéance 1987-89

180,0

Bons $9,125 \%$ à 3 ans, échéance 1984

150,0

Bons $7,375 \%$ à 2 ans, échéance 1984

150,0 
Autres placements

Bons 7,75\%, échéance 1986

Prêt 9\%, échéance 1986

100,0

Bons 8,50\%, échéance 1987

200,0

Bons 7,50\%, échéance 1988

300,0

Bons 7,25\%, échéance 1987

300,0

Bons 6,5\%, échéance 1985

78,0

Prêt 7,25\%, échéance 1988

200,0

Bons 6,75\%, échéance 1987

300,0

Obligations 6,375\%, échéance 1991

80,0

TOTAL

$3.040,0$

Source : Banque mondiale, rapport annuel 1982.

\section{ASSEMBLEE ANNUELLE DU CAD}

Le CAD constate qu'en 1980 les pays de l'OCDE ont encore fourni trop peu d'aide publique au développement; il réclame une utilisation plus efficace des ressources, ainsi que des priorités régionales et sectorielles. Pour 1981, il relève même une baisse des prestations publiques en faveur de l'aide au développement et l'on regrette le faible niveau des contributions de la Suisse.

Le 18 novembre 1981, le Comité d'aide au développement de I'OCDE (CAD) tint sa 20ème assemblée annuelle. Ministres et directeurs des organisations nationales de coopération au développement se réunirent pour un échange d'opinions sur les problèmes majeurs de la politique de développement dans le cadre de l'actuelle politique économique. Font partie du comité les représentants de dix-huit pays industriels et de la commission de la CEE. La Suisse en est devenue membre en 1968.

La tâche du CAD consiste à faciliter les travaux de coopération au développement des pays de l'OCDE et à en évaluer le volume, les conditions, les formes, la distribution géographique et sectorielle. II examine les prestations de chaque pays membre et tente de déterminer les critères qualitatifs et quantitatifs de l'aide, tout en s'efforçant d'en améliorer la coordination et l'efficacité.

Lors de cette 20ème assemblée, on a d'abord étudié l'ensemble du "transfert de développement" attendu (les prestations de l'économie privée, humanitaires, publiques et multilatérales dans leur globalité). Vu la détérioration de la conjoncture, on ne saurait s'attendre qu'à une légère progression des prestations. La somme du transfert de développement de l'ensemble des pays de I'OCDE s'est élevée en 1980 à 1,04\% du PNB, l'aide publique au développe- 
ment ne représentant que $0,37 \%$ du PNB.

Le CAD estime qu'au cours des cinq années à venir, I'aide publique au développement ne progressera chaque année que de 2 à $3 \%$ en moyenne, en chiffres réels. La part du PNB représentée par l'apport de fonds publics à I'aide au développement ne devrait s'améliorer que de 0,01\% et atteindre ainsi $0,38 \%$. On reste donc fort loin du but : consacrer à la coopération au développement $0,7 \%$ du PNBdes pays donateurs.

De tous les aspects du transfert de développement, on discuta surtout des apports de l'économie privée, sans doute à cause de l'importance que revêt cette forme d'intervention dans l'actuelle politique américaine de développement. On parla également à ce sujet des crédits mixtes, en forte augmentation ces derniers temps. A noter en 1980 le léger recul des apports financiers fournis aux pays en développement par l'économie privée des pays de I'OCDE.

La coopération au développement continuant d'être chichement financée, il importe que l'aide publique au développement soit d'autant plus efficace et mieux axée sur les tâches prioritaires. A mettre au premier plan une concentration régionale sur les pays en développement les plus pauvres et une concentration sectorielle sur l'alimentation, l'énergie, les ressources humaines et la protection de l'environnement. A retenir aussi la nécessité d'une meilleure évaluation des divers projets, d'un dialogue plus soutenu entre pays donateurs et pays bénéficiaires, au niveau multilatéral et dans le cadre des institutions de coordination existantes. On envisage aussi de conclure des contrats de développement qui réglementeraient les droits et les devoirs des Etats liés par un effort de coopération au développement.

La possibilité de procurer de nouvelles ressources financières à la coopération internationale au développement a été évoquée en envisageant l'instauration d'une garantie multilatérale des investissements pour la couverture des risques non commerciaux (politiques). De même, le problème des crédits multilatéraux qui entrent en concurrence avec I'aide publique bilatérale a été mentionné.

Dirigée par l'ambassadeur Heimo, directeur de la DDA, la délégation suisse reconnut que les apports en fonds publics de la coopération suisse au développement étaient modestes, mais qu'on s'attendait à les voir progresser d'année en année. La demande accrue de crédits mixtes de la part de l'aide publique au développement est intéressante, car elle est susceptible de mobiliser de nouveaux apports de l'économie privée en faveur des pays en développement. Au demeurant, les crédits mixtes devraient être utilisés selon les critères généralement en usage dans la pratique du développement. En 1980, la Suisse a consacré $2,6 \%$ (soit 4,17 milliards de francs) de son PNB au transfert de développement, mais la part des prestations en fonds publics n'a atteint que $0,24 \%$ du PNB (82).

Lors d'une réunion extraordinaire, fin juin 1982, le CAD a fait connaître le 
montant du transfert de développement en 1981. Le total des prestations a légèrement augmenté et s'est élevé en 1981 à 1,13\% du PNB de l'ensemble des Etats de I'OCDE. L'aide publique au développement est par contre en recul. Elle n'a plus représenté en 1981 que 0,35\% du PNB de l'ensemble des pays de l'OCDE, à commencer par les Etats-Unis dont les prestations en faveur de l'aide au développement ont reculé de $26 \%$. En chiffres absolus, la totalité du transfert de développement s'est élevée en 1981 à 81,4 milliards de dollars EU, I'aide publique au développement à 25,46 milliards de dollars EU.

Examinant les prestations des divers pays de l'OCDE, l'assemblée constata que l'aide publique au développement de la Suisse en 1981 était restée stagnante aux environs de $0,24 \%$ du PNB. Ce qui vaut à la Suisse l'antépénultième rang, parmi les pays de l'OCDE. Le CAD regretta que les mesures d'économie prises par la Confédération eussent touché plus durement en pourcentage l'aide publique au développement que tous les autres postes du budget. Une augmentation de l'aide publique au développement dans le cadre prévu n'est guère possible (83). 